\title{
Finding the Cost-Optimal Path with Time Constraint over Time-Dependent Graphs
}

\author{
Yajun Yang ${ }^{1,2}$, Hong Gao ${ }^{3}$, Jeffrey $\mathrm{Xu} \mathrm{Yu}{ }^{4}$, Jianzhong $\mathrm{Li}^{3}$ \\ ${ }^{1}$ School of Computer Science and Technology, Tianjin University, China \\ ${ }^{2}$ Tianjin Key Laboratory of Cognitive Computing and Application, Tianjin, China \\ ${ }^{3}$ School of Computer Science and Technology, Harbin Institute of Technology, China \\ ${ }^{4}$ The Chinese University of Hong Kong, China \\ yjyang@tju.edu.cn, honggao@hit.edu.cn, yu@se.cuhk.edu.hk, lijzh@hit.edu.cn
}

\begin{abstract}
Shortest path query is an important problem and has been well studied in static graphs. However, in practice, the costs of edges in graphs always change over time. We call such graphs as timedependent graphs. In this paper, we study how to find a costoptimal path with time constraint in time-dependent graphs. Most existing works regarding the Time-Dependent Shortest Path (TD$\mathrm{SP})$ problem focus on finding a shortest path with the minimum travel time. All these works are based on the following fact: the earliest arrival time at a vertex $v$ can be derived from the earliest arrival time at $v$ 's neighbors. Unfortunately, this fact does not hold for our problem. In this paper, we propose a novel algorithm to compute a cost-optimal path with time constraint in time-dependent graphs. We show that the time and space complexities of our algorithm are $O(k n \log n+m k)$ and $O((n+m) k)$ respectively. We confirm the effectiveness and efficiency of our algorithm through conducting experiments on real datasets with synthetic cost.
\end{abstract}

\section{INTRODUCTION}

Shortest path query is an important problem in graphs and has been well studied in static graphs. However, graphs often evolve over time. For example, the Vehicle Information and Communication System (VICS) and the European Traffic Message Channel (TMC) are two transportation systems, which can provide real-time traffic information to users. Such transportation networks are timedependent graphs, i.e., the travel time for a road varies with time taking "rush hour" into account. Meanwhile, the toll fee of a road is also time-dependent. For example, there are "London congestion charge" and "Road pricing policy" to reduce traffic congestion and control traffic pollution in the United Kingdom [14]. The vehicles are charged if they pass through the major roads in rush hours. The similar policies are also applied in Singapore. This results in the variation of the toll fee of a road in different hours of a day and different days of a week, which shows the toll fee is time-dependent. Moreover, there are several works that study the pricing mechanism for the time-dependent toll fee $[15,12]$.

This work is licensed under the Creative Commons AttributionNonCommercial-NoDerivs 3.0 Unported License. To view a copy of this license, visit http://creativecommons.org/licenses/by-nc-nd/3.0/. Obtain permission prior to any use beyond those covered by the license. Contact copyright holder by emailing info@vldb.org. Articles from this volume were invited to present their results at the 40th International Conference on Very Large Data Bases, September 1st - 5th 2014, Hangzhou, China.

Proceedings of the VLDB Endowment, Vol. 7, No. 9

Copyright 2014 VLDB Endowment 2150-8097/14/05.
Consider an application in a road network. Someone has an appointment with her friends. The earliest departure time for her is $t_{1}$ and she has to arrive at rendezvous before time $t_{2}$. In this road network, there are two kinds of costs for every road, travel time and toll fee, which are both time-dependent. According to the travel time, it can be verified whether a path $p$ satisfies time constraint or not, i.e., whether one can arrive before time $t_{2}$ along path $p$. It is worth noting that there may be several paths from the source to the destination satisfying the time constraint. Therefore, it is very important to find an optimal path with the minimum cost from all the paths satisfying the time constraint.

In the above example, the road network can be considered as a large graph $G$ with time information. Every edge $\left(v_{i}, v_{j}\right)$ in $G$ has two kinds of costs: $w_{i, j}(t)$ and $f_{i, j}(t) . w_{i, j}(t)$ is the time cost to specify how long it takes to travel through an edge $\left(v_{i}, v_{j}\right)$, and $f_{i, j}(t)$ is the toll fee for traveling through an edge $\left(v_{i}, v_{j}\right)$. Both $w_{i, j}(t)$ and $f_{i, j}(t)$ are the functions that are dependent on the departure time $t$ at the starting endpoint $v_{i}$ of the edge $\left(v_{i}, v_{j}\right)$. We call such graphs time-dependent graphs. The query of a costoptimal path with time constraint in time-dependent graphs can be defined as follows. Given a source $v_{s}$, a destination $v_{e}$, the earliest departure time $t_{d}$ and the latest arrival time $t_{a}$, find an optimal path $p$ from $v_{s}$ to $v_{e}$, satisfying the following two conditions: (1) departing from $v_{s}$ after time $t_{d}$, one can arrive at $v_{e}$ before time $t_{a}$ along path $p$; and (2) path $p$ has the minimum cost (toll fee) among all the paths satisfying the condition (1).

There are many works on the shortest path problem in timedependent graphs $[13,7]$. Most of them are to find an optimal path with the minimum travel time from the source to the destination, when the departure time from the source can be selected from a user-given starting-time interval. These works assume there is only one time function $w_{i, j}(t)$ on every edge in a time-dependent graph. Let $\lambda_{i}$ denote the earliest arrival time at vertex $v_{i} . \lambda_{i}$ can be calculated by the following equation:

$$
\lambda_{i}=\min \left\{\left(\lambda_{j}+\omega\left(v_{j}\right)\right)+w_{j, i}\left(\lambda_{j}+\omega\left(v_{j}\right)\right) \mid v_{j} \in N^{-}\left(v_{i}\right)\right\}
$$

where $v_{j} \in N^{-}\left(v_{i}\right)$ represents that $v_{j}$ is an incoming neighbor of $v_{i}$ and $\omega\left(v_{j}\right)$ is the waiting time at $v_{j}$. This equation indicates that the earliest arrival time at a vertex can be obtained based on the earliest arrival time at this vertex's incoming neighbors. All these works on the TDSP problem utilize this property to compute the shortest paths with the minimum travel time. Unfortunately, this property does not hold for our problem (detailed in Section 2.2). Thus, the existing works on the TDSP problem cannot solve our problem proposed in this paper.

In this paper, we study the problem of identifying a cost-optimal path with time constraint in time-dependent graphs. Different to 
the TDSP problem, we consider two kinds of costs for every edge in this paper. To the best of our knowledge, our work is the first one regarding this problem under the continuous time model. The main contributions are summarized below. First, we propose a novel algorithm to find a cost-optimal path with time constraint in timedependent graphs. Our algorithm can handle both undirected and directed time-dependent graphs. Second, we show that the time and space complexities of our algorithm are $O(k n \log n+m k)$ and $O((n+m) k)$ respectively, where $n$ is the number of vertices, and $m$ is the number of edges, and $k$ is the average number of piecewise constant values of the atmc-function (detailed in Section 3.1). Third, we confirm the effectiveness and efficiency of our algorithm through conducting experiments on real datasets. Compared with the state of the art algorithm used for discrete time model, our algorithm not only can find the optimal path but also makes at least two orders of magnitude improvement in time and space overhead.

The rest of the paper is organized as follows. Section 2 defines the problem. Section 3 proposes a two-step algorithm to find a cost-optimal path with time constraint. The experiment results are presented in Section 4. The related works are introduced in Section 5. We conclude this paper in Section 6.

\section{PROBLEM STATEMENT}

\subsection{Time-Dependent Graph and Cost-Optimal Path with Time Constraint}

Definition 2.1: (Time-Dependent Graph) A time-dependent graph is a simple graph, denoted as $G_{T}(V, E, W, F)$ (or $G_{T}$ for short), where $V=\left\{v_{i}\right\}$ is the set of vertices; $E \subseteq V \times V$ is the set of edges; $W$ and $F$ are two sets of non-negative value functions. For every edge $\left(v_{i}, v_{j}\right) \in E$, there are two functions: time-function $w_{i, j}(t) \in W$ and cost-function $f_{i, j}(t) \in F$, where $t$ is a time variable. A time function $w_{i, j}(t)$ specifies how much time it takes to travel from $v_{i}$ to $v_{j}$, if departing from $v_{i}$ at time $t$. A cost function $f_{i, j}(t)$ specifies how much cost (e.g., toll fee) it takes to travel from $v_{i}$ to $v_{j}$, if departing from $v_{i}$ at time $t$.

In this paper, we assume that $w_{i, j}(t) \geq 0$ and $f_{i, j}(t) \geq 0$. The assumption is reasonable, because the travel time and travel cost cannot be less than zero in real applications. Our work can be easily extended to handle undirected graphs, in which an undirected edge $\left(v_{i}, v_{j}\right)$ is equivalent to two directed edges $\left(v_{i}, v_{j}\right)$ and $\left(v_{j}, v_{i}\right)$, where $w_{i, j}(t)=w_{j, i}(t)$ and $f_{i, j}(t)=f_{j, i}(t)$. For simplicity, we only consider directed graphs in the rest of this paper.

We assume that cost-function $f_{i, j}(t)$ is a piecewise constant function, which can be formalized as follows:

$$
f_{i, j}(t)= \begin{cases}c_{1}, & t^{0} \leq t<t^{1} \\ c_{2}, & t^{1} \leq t<t^{2} \\ \cdots & \\ c_{p}, & t^{\sigma-1} \leq t \leq t^{\sigma}\end{cases}
$$

Here, $\left[t^{0}, t^{\sigma}\right]$ is the time domain of function $f_{i, j}(t) . c_{x} \quad(1 \leq x \leq$ $\sigma)$ is a constant value, which represents the value of $f_{i, j}(t)$ when $t \in\left[t^{x-1}, t^{x}\right]$. The assumption is reasonable. In real applications, the cost functions are always piecewise constant. For example, in road networks, the toll fees for traveling through a road are distinct constant values during day and night. It means the cost-function of this road is a piecewise constant function.

Given a path $p$, the cost of $p$ is also time dependent. For any edge $\left(v_{i}, v_{j}\right) \in p$, if the departure time from $v_{i}$ is different, the travel cost for edge $\left(v_{i}, v_{j}\right)$ is different too. In order to find a costoptimal path, some waiting time is allowed, denoted as $\omega\left(v_{i}\right)$, at
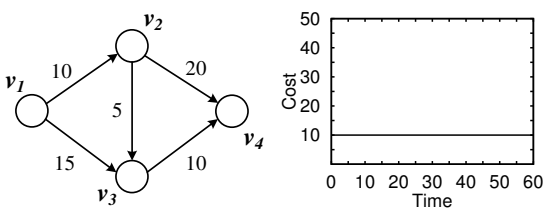

(b) $f_{1,2}(t)$

(a) $(V, E, W)$
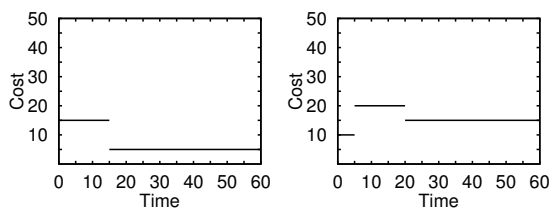

(d) $f_{2,3}(t)$

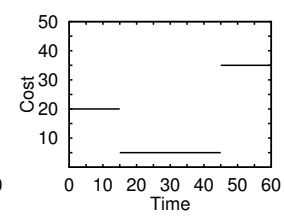

(c) $f_{1,3}(t)$

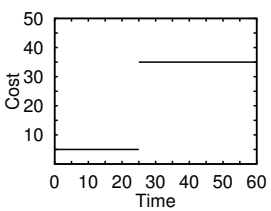

(f) $f_{3,4}(t)$
Figure 1: Time-dependent graph

each vertex $v_{i}$. That is, when arriving at vertex $v_{i}$, one can wait a time period $\omega\left(v_{i}\right)$ if the cost of path $p$ can be minimized. We use arrive $\left(v_{i}\right)$ and depart $\left(v_{i}\right)$ to denote the arrival time at $v_{i}$ and departure time from $v_{i}$, respectively. For each vertex $v_{i}$, we have

$$
\operatorname{depart}\left(v_{i}\right)=\operatorname{arrive}\left(v_{i}\right)+\omega\left(v_{i}\right)
$$

Let $p=v_{1} \rightarrow v_{2} \rightarrow \cdots \rightarrow v_{h}$ be a given path with the earliest departure time $t_{d}$ and the waiting time $\omega\left(v_{i}\right)$ for each vertex $v_{i}$, then we have

$$
\begin{aligned}
\operatorname{arrive}\left(v_{1}\right) & =t_{d} \\
\operatorname{arrive}\left(v_{2}\right) & =\operatorname{depart}\left(v_{1}\right)+w_{1,2}\left(\operatorname{depart}\left(v_{1}\right)\right) \\
\ldots & \\
\operatorname{arrive}\left(v_{h}\right) & =\operatorname{depart}\left(v_{h-1}\right)+w_{h-1, h}\left(\operatorname{depart}\left(v_{h-1}\right)\right)
\end{aligned}
$$

For any vertex $v_{i} \in p$, we use $\operatorname{cost}_{p}\left(v_{i}\right)$ to denote the cost from $v_{1}$ to $v_{i}$ by path $p \cdot \operatorname{cost}_{p}\left(v_{i}\right)$ can be calculated recursively as follows:

$$
\begin{aligned}
\operatorname{cost}_{p}\left(v_{1}\right) & =0 \\
\operatorname{cost}_{p}\left(v_{2}\right) & =\operatorname{cost}_{p}\left(v_{1}\right)+f_{1,2}\left(\operatorname{depart}\left(v_{1}\right)\right) \\
\ldots & \\
\operatorname{cost}_{p}\left(v_{h}\right) & =\operatorname{cost}_{p}\left(v_{h-1}\right)+f_{h-1, h}\left(\operatorname{depart}\left(v_{h-1}\right)\right)
\end{aligned}
$$

The cost of path $p$ is defined as $\operatorname{cost}(p)=\operatorname{cost}_{p}\left(v_{h}\right)$. Next, we give the definition of the problem of the cost-optimal path with time constraint in time-dependent graphs.

Definition 2.2: (Cost-Optimal Path with Time Constraint) Given a time-dependent graph $G_{T}$, a source vertex $v_{s}$, a destination vertex $v_{e}$, the earliest departure time $t_{d}$ and the latest arrival time $t_{a}$, the problem of the cost-optimal path with time constraint is to find an optimal path $p^{*}$ and the optimal waiting time $\omega^{*}\left(v_{i}\right)\left(\omega^{*}\left(v_{i}\right)\right.$ $\geq 0)$ for every vertex $v_{i} \in p^{*}$, such that (1) $\operatorname{depart}\left(v_{s}\right) \geq t_{d} \wedge$ arrive $\left(v_{e}\right) \leq t_{a}$; and (2) $\operatorname{cost}\left(p^{*}\right)$ is the minimum among all the paths from $v_{s}$ to $v_{e}$ that satisfy the condition (1).

Fig. 1 illustrates an example of time-dependent graph $G_{T}$. Here, Fig. 1(a) presents the structure of $G_{T}$ and the travel time $w_{i, j}(t)$ for every edge $\left(v_{i}, v_{j}\right) \in G_{T}$. In this example, the travel time for every edge is a constant value. The cost-functions $f_{i, j}(t)$ for five edges, $\left(v_{1}, v_{2}\right),\left(v_{1}, v_{3}\right),\left(v_{2}, v_{3}\right),\left(v_{2}, v_{4}\right)$ and $\left(v_{3}, v_{4}\right)$ are shown in Fig. 1(b), (c), (d), (e) and (f), respectively.

Given a query of the cost-optimal path with time constraint: $v_{s}=$ $v_{1}, v_{e}=v_{4}, t_{d}=0$ and $t_{a}=60$, a cost-optimal path is $p^{*}=$ $v_{1} \rightarrow v_{2} \rightarrow v_{3} \rightarrow v_{4}$. The optimal waiting time are $\omega^{*}\left(v_{1}\right)=0$, $\omega^{*}\left(v_{2}\right)=5$, and $\omega^{*}\left(v_{3}\right)=0$, respectively. The cost of the optimal path $p^{*}$ is $\operatorname{cost}\left(p^{*}\right)=20$. 


\subsection{Existing Solutions for the TDSP Problem}

Most existing works for the TDSP problem are to find an optimal path with the minimum travel time. We discuss two most recently published efficient algorithms for the TDSP problem and give the reasons that why they cannot be used to solve our problem.

A* Algorithm: Kanoulas et al. in [13] propose an extension to $\mathrm{A}^{*}$ algorithm for the TDSP problem. The main idea is to estimate a lower bound of travel time from source to destination and expand path utilizing this lower bound. The main problems of this $\mathrm{A}^{*}$-extended algorithm are summarized below. (1) This algorithm needs to compute the Euclidean distance between any two vertices and the maximum speed in a road network to estimate the lower bound by the equation "distance ". However, for our problem, travel cost cannot be estimated by this equation. Therefore, this algorithm cannot be used to our problem. (2) The efficiency of this algorith$\mathrm{m}$ is dependent on the pruning power of the estimation of travel time. This algorithm is efficient when source and destination are close to each other in a graph. It is difficult to figure out such an estimation in a large graph. When a graph is large or source is far away from destination, the algorithm is inefficient. (3) In the worst case, all paths from source to destination are enumerated and maintained, and then the time and space complexities of this algorithm is exponential w.r.t. the size of $G_{T}$.

2S Algorithm: Ding et al. in [7] propose a more efficient algorithm to address the TDSP problem. This algorithm includes two phases: (1) time-refinement phase; and (2) path-selection phase. In the first phase, the algorithm refines the earliest arrival time for every vertex $v_{i}$ by the following equation:

$$
g_{i}(t)=\min _{v_{j} \in N^{-}\left(v_{i}\right), \omega\left(v_{j}\right)}\left\{\left(g_{j}(t)+\omega\left(v_{j}\right)\right)+w_{j, i}\left(g_{j}(t)+\omega\left(v_{j}\right)\right)\right\}
$$

Here, $g_{i}(t)$ is the earliest arrival time for $v_{i}$, if departing from source $v_{s}$ at starting time $t . N^{-}\left(v_{i}\right)$ is $v_{i}$ 's incoming neighbor set, i.e., $N^{-}\left(v_{i}\right)=\left\{v_{j} \mid\left(v_{j}, v_{i}\right) \in E\right\}$. The algorithm utilizes a priority queue $Q$ to maintain the earliest arrival time function $g_{i}(t)$ and a time interval $\left[t_{s}, \tau_{i}\right]$ for all the vertices in $G_{T}$. The value of $g_{i}(t)$ for $t \in\left[t_{s}, \tau_{i}\right]$ is corrected. In each iteration, a vertex $v_{i}$ with the minimum $g_{i}\left(\tau_{i}\right)$ is dequeued from $Q$. The algorithm refines $g_{i}(t)$ and $\left[t_{s}, \tau_{i}\right]$ by Eq. (1). Let $I$ denote the user-given starting time interval. If $\left[t_{s}, \tau_{i}\right] \neq I$, then $v_{i}$ is inserted into $Q$ again. The algorithm terminates when the earliest arrival time function $g_{e}(t)$ of destination $v_{e}$ has been refined in the whole time interval $I$.

The main problem of the $2 \mathrm{~S}$ algorithm is that this algorithm needs to compute the earliest arrival time function by Eq. (1). However, the rationale Eq. (1) based on does not hold for the costoptimal path problem proposed in this paper. We clarify this point using the example in Fig. 1. Suppose our objective is to find a costoptimal path from $v_{1}$ to $v_{4}$. For an incoming neighbor $v_{3}$ of vertex $v_{4}$, we find the minimum cost from $v_{1}$ to $v_{3}$ is $g_{3}(15)=5$, i.e., The cost of path $v_{1} \rightarrow v_{3}$ is the minimum when departing from source $v_{1}$ at time $t=15$. In this case, the arrival time at $v_{3}$ is 30 . In the other words, the earliest departure time from $v_{3}$ is 30 if one arrives at $v_{3}$ with cost $g_{3}(15)=5$. By Eq. (1), the minimum cost from $v_{1}$ to $v_{4}$ by edge $\left(v_{3}, v_{4}\right)$ is $g_{3}(15)+w_{3,4}(30)=5+35=40$. However, the cost-optimal path from $v_{1}$ to $v_{4}$ via $v_{3}$ is $v_{1} \rightarrow v_{2} \rightarrow$ $v_{3} \rightarrow v_{4}$, and its cost is 20. In this path, the cost from $v_{1}$ to $v_{3}$ is 15 , which is larger than the minimum cost $g_{3}(15)=5$. This example shows that a sub-path of the cost-optimal path may not be a costoptimal path. In the example, the cost-optimal path from $v_{1}$ to $v_{4}$ is $v_{1} \rightarrow v_{2} \rightarrow v_{3} \rightarrow v_{4}$, but its sub-path $v_{1} \rightarrow v_{2} \rightarrow v_{3}$ is not a costoptimal path from $v_{1}$ to $v_{3}$. Let $p_{s \rightsquigarrow j}$ denote the cost-optimal path from source $v_{s}$ to $v_{j}$ and $t_{j}$ denote the arrival time at $v_{j}$ along path $p_{s \rightsquigarrow j}$. Note that there may exist another path $p_{s \rightsquigarrow j}^{\prime}$ from $v_{s}$ to $v_{j}$.

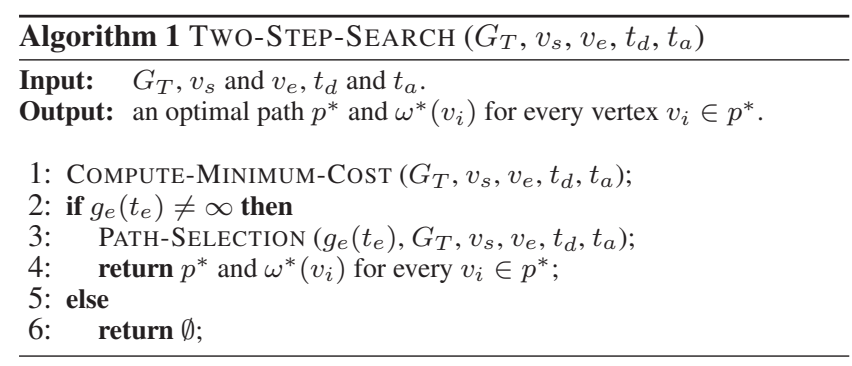

Similarly, $t_{j}^{\prime}$ is the arrival time at $v_{j}$ along path $p_{j}^{\prime}$. The cost of path $p_{s \rightsquigarrow j}^{\prime}$ is slightly larger than that of $p_{s \rightsquigarrow j}$. However, the minimum cost to travel through edge $\left(v_{j}, v_{i}\right)$ after time $t_{j}$ is far more than after time $t_{j}^{\prime}$, i.e., $\min \left\{w_{j, i}(t) \mid t \geq t_{j}\right\} \gg \min \left\{w_{j, i}(t) \mid t \geq t_{j}^{\prime}\right\}$. Thus, $p_{s \rightsquigarrow j \rightarrow i}$ is not a cost-optimal path from $v_{s}$ to $v_{i}$. It means the minimum arrival cost at a vertex $v_{i}$ cannot be computed based on the minimum arrival costs at $v$ 's incoming neighbors, which is indicated by Eq. (1). It is because that a sub-path of a cost-optimal path may not be the cost-optimal path.

Several studies in the field of operation research consider the optimal path problem under the discrete time model $[3,2]$. In the discrete time model, a whole time interval is discretized to a set of time points, $\left\{t_{1}, t_{2}, \cdots, t_{l}\right\}$. For any edge $\left(v_{i}, v_{j}\right)$, only one specified time point $t_{x}$ to depart from $v_{i}$ can be selected. The main disadvantages of these works are as follows: (1) An optimal path may not be found under the discrete time model. Suppose one arrives at vertex $v_{i}$ at time $t, t_{i-1}<t<t_{i}$, where $t_{i-1}$ and $t_{i}$ are two consecutive time points in the given set of discrete time points. The earliest departure time from $v_{i}$ is $t_{i}$. However, the best departure time is $t^{\prime}$, because the cost to travel through edge $\left(v_{i}, v_{j}\right)$ is the minimum if one departs from $v_{i}$ at time $t^{\prime}$. Here, $t<t^{\prime}<t_{i}$. (2) These works need to compute the arriving cost for every vertex at every time point. The time and space costs are expensive.

\section{TWO-STEP ALGORITHM}

We propose an efficient TWO-STEP-SEARCH algorithm. We first introduce what is the "arrival-time and the minimum-cost function" (or atmc-function for simplicity) and how to compute atmcfunction for a vertex $v_{i}$ in $G_{T}$. Second, we introduce the first step of the TWO-STEP-SEARCH algorithm, i.e., how to compute the minimum cost from source $v_{s}$ to destination $v_{e}$. Third, we introduce the second step of the TWO-STEP-SEARCH algorithm, i.e., how to find a cost-optimal path and the optimal waiting time for every vertex in the optimal path. Finally, we discuss the time complexity and space complexity. The TWO-STEP-SEARCH algorithm is shown in Algorithm 1.

In the following, we first focus on the case where the travel time for every edge $\left(v_{i}, v_{j}\right)$ in $G_{T}$ is a constant value $w_{i, j}$, i.e., $w_{i, j}(t)=w_{i, j}$. We will discuss how to deal with the general case where the travel time for every edge is a function $w_{i, j}(t)$ in Section 3.5. $w_{i, j}(t)$ is related to the departure time $t$ from $v_{i}$.

\subsection{Arrival-Time and Min-Cost Function}

Given a time-dependent graph $G_{T}$, for any vertex $v_{i} \in G_{T}$, there may exist several paths that depart from source $v_{s}$ after time $t_{d}$ and arrive at $v_{i}$ at time point $t$. Let $P_{i}(t)$ denote the set of all such paths and $g_{i}(t)$ denote the minimum cost among all the paths in $P_{i}(t)$, that is,

$$
g_{i}(t)=\min \left\{\operatorname{cost}(p) \mid p \in P_{i}(t)\right\}
$$

Note that some waiting time is allowed, as long as a path $p$ satisfies the time constraint: (1) $\operatorname{depart}_{p}\left(v_{s}\right) \geq t_{d}$, it means that one departs 
Table 1: Important notations

\begin{tabular}{|c|c|}
\hline Notation & Description \\
\hline$G_{T}, v_{s}, v_{e}$ & time-dependent graph, source, destination \\
\hline$t_{d}, t_{a}$ & earliest departure time, latest arrival time \\
\hline$f_{i, j}(t), w_{i, j}(t)$ & cost-function, time-function \\
\hline$g_{i}(t)$ & atmc-function of vertex $v_{i}$ \\
\hline$g_{j \rightarrow i}(t)$ & atmc-function by edge $\left(v_{j}, v_{i}\right)$ \\
\hline$\lambda_{j}$ & earliest arrival time at $v_{j}$ \\
\hline
\end{tabular}

from source $v_{s}$ after time $t_{d}$ by path $p$; and (2) $\operatorname{arrive}_{p}\left(v_{i}\right)=t$, it means that one arrives at $v_{i}$ at time $t$ by path $p . g_{i}(t)$ is a function related to arrival time $t$ for $v_{i}$. We call $g_{i}(t)$ the atmc-function of vertex $v_{i} \cdot g_{i}(t)$ represents the minimum cost that one can arrive at $v_{i}$ at time $t$ from source $v_{s}$.

Given a time-dependent graph $G_{T}$, if the cost function $f_{i, j}(t)$ is a piecewise constant function for every edge $\left(v_{i}, v_{j}\right)$ in $G_{T}$, then for any vertex $v_{i} \in V$, it is obvious that $g_{i}(t)$ of $v_{i}$ is also a piecewise constant function.

Based on the atmc-function, the minimum cost from $v_{s}$ to $v_{e}$ can be defined as $g_{e}\left(t_{e}\right)=\min \left\{g_{e}(t) \mid t \in\left[\lambda_{e}, t_{a}\right]\right\}$, where $t_{e}$ is a time point that minimizes $g_{e}(t)$ for $t \in\left[\lambda_{e}, t_{a}\right]$ and $\lambda_{e}$ represents the earliest arrival time for $v_{e}$ if departing from $v_{s}$ after the earliest departure time $t_{d}$.

The main idea in the first step of the TwO-STEP-SEARCH algorithm is to update atmc-function $g_{i}(t)$ iteratively for every vertex $v_{i} \in G_{T}$ until the minimum cost from source $v_{s}$ to destination $v_{e}$ is derived. Note that in each iteration, the current $g_{i}(t)$ of $v_{i}$ may not be an optimal (or correct) atmc-function. We need to update $g_{i}(t)$ using its incoming neighbors' atmc-functions such that $g_{i}(t)$ is closer to the optimal atmc-function.

Next, we discuss how to update $g_{i}(t)$ for a vertex $v_{i}$. Let $v_{j}$ be an incoming neighbor of $v_{i}$, i.e., $v_{j} \in N^{-}\left(v_{i}\right)$. Suppose $g_{j}(t)$ is the current atmc-function of $v_{j}$. The updating process includes two phases: (1) compute $g_{j \rightarrow i}(t), g_{j \rightarrow i}(t)$ is a function as similar as atmc-function, which represents the minimum cost that one departing from $v_{s}$ can arrive at $v_{i}$ at time point $t$ by edge $\left(v_{j}, v_{i}\right)$; and (2) update $g_{i}(t)$ using $g_{j \rightarrow i}(t)$.

If the waiting time is not allowed, i.e., $\omega\left(v_{j}\right)=0$, we have:

$$
g_{j \rightarrow i}(t)=g_{j}\left(t-w_{j, i}\right)+f_{j, i}\left(t-w_{j, i}\right)
$$

The meaning of Eq. (2) is that: if the arrival time for vertex $v_{i}$ is $t$, then one need to depart from $v_{j}$ at time $t-w_{j, i}$. Because there is no waiting time, the arrival time for $v_{j}$ is $t-w_{j, i} \cdot g_{j}\left(t-w_{j, i}\right)$ is the minimum cost from $v_{s}$ to $v_{j}$ for arrival time $t-w_{j, i}$ and $f_{j, i}\left(t-w_{j, i}(t)\right)$ is the cost to travel edge $\left(v_{j}, v_{i}\right)$, then $g_{j \rightarrow i}(t)$ is the sum of $g_{j}\left(t-w_{j, i}\right)$ and $f_{j, i}\left(t-w_{j, i}(t)\right)$.

In this paper, some waiting time is allowed, then we have

$$
\begin{aligned}
g_{j \rightarrow i}(t) & =\min _{t^{\prime}, \omega\left(v_{j}\right)}\left\{g_{j}\left(t^{\prime}\right)+f_{j, i}\left(t^{\prime}+\omega\left(v_{j}\right)\right)\right\} \\
t & =t^{\prime}+\omega\left(v_{j}\right)+w_{j, i}
\end{aligned}
$$

Here, $t^{\prime}+\omega\left(v_{j}\right)$ is the departure time from $v_{j}$ and $t^{\prime}$ is the arrival time for $v_{j}$. It means after arriving at $v_{j}$, one needs to wait for $\omega\left(v_{j}\right)$ time before departure from $v_{j}$. To guarantee that the arrival time for $v_{i}$ is $t$, an appropriate waiting time is necessary to satisfy that $t=t^{\prime}+\omega\left(v_{j}\right)+w_{j, i}$. Therefore, to compute $g_{j \rightarrow i}(t)$ is equivalent to find the optimal arrival time $t^{\prime}$ and the waiting time $\omega\left(v_{j}\right)$, which satisfies $t=t^{\prime}+\omega\left(v_{j}\right)+w_{j, i}$, such that $g_{j \rightarrow i}(t)$ is minimized in Eq. (3).

Given the arrival time $t$ for vertex $v_{i}$, the departure time from $v_{j}$ is fixed to $t-w_{j, i}$. Then, the cost to travel the edge $\left(v_{j}, v_{i}\right)$ is $f_{j, i}\left(t-w_{j, i}\right)$. By the definition of the function $g_{j \rightarrow i}(t)$, we have the following equation:

$$
\begin{aligned}
g_{j \rightarrow i}(t) & =\min _{t^{\prime} \leq t-w_{j, i}}\left\{g_{j}\left(t^{\prime}\right)+f_{j, i}\left(t-w_{j, i}\right)\right\} \\
& =\min _{t^{\prime} \leq t-w_{j, i}}\left\{g_{j}\left(t^{\prime}\right)\right\}+f_{j, i}\left(t-w_{j, i}\right)
\end{aligned}
$$

By Eq. (4), we find that computing $g_{j \rightarrow i}(t)$ is equivalent to find the optimal time $t^{\prime}\left(t^{\prime} \leq t-w_{j, i}\right)$, such that $g_{j}\left(t^{\prime}\right)$ is the minimum. In the other words, for any departure time point $t-w_{j, i}$, we only need to find the minimum $g_{j}\left(t^{\prime}\right)$ for $t^{\prime} \leq t-w_{j, i}$ to minimize the sum of $g_{j}\left(t^{\prime}\right)$ and $f_{j, i}\left(t-w_{j, i}\right)$.

We use $\lambda_{j}$ to denote the earliest arrival time at vertex $v_{j}$ if departing from $v_{s}$ at the earliest departure time $t_{d}$. It indicates that one cannot arrive at $v_{j}$ before $\lambda_{j}$ if departing from $v_{s}$ at (or after) time $t_{d}$. Thus, the time domain of $g_{j}(t)$ is $\left[\lambda_{j}, t_{a}\right]$. Similarly, the time domain of $g_{j \rightarrow i}(t)$ is $\left[\lambda_{j \rightarrow i}, t_{a}\right]$, where $\lambda_{j \rightarrow i}=\lambda_{j}+w_{j, i}$. $\lambda_{j \rightarrow i}$ is the earliest time that one can arrive at $v_{i}$ by edge $\left(v_{j}, v_{i}\right)$ if departing from $v_{s}$ at time $t_{d}$.

The procedure to compute $g_{j \rightarrow i}(t)$ is as follows. We find the minimum $g_{j}\left(t^{\prime}\right)$ iteratively to minimize $g_{j \rightarrow i}(t)$ in Eq. (4) until $g_{j \rightarrow i}(t)$ is computed for $t \in\left[\lambda_{j \rightarrow i}, t_{a}\right]$. To compute $g_{j \rightarrow i}(t)$, the whole time interval of $g_{j}(t)$ is set as $T_{j, i}=\left[\lambda_{j}, t_{a}-w_{j, i}\right]$, because one cannot arrive at $v_{i}$ before time $t_{a}$ if departing from $v_{j}$ after $t_{a}-w_{j, i}$. We use $S_{j, i}$ to denote the processed time interval for $g_{j}(t)$ in each iteration. It means that the minimum $g_{j}\left(t^{\prime}\right)$ in Eq. (4) has been found to minimize $g_{j \rightarrow i}(t)$ for $t \in S_{j, i} \oplus w_{j, i}$. Here, $S_{j, i} \oplus w_{j, i}$ represents the time interval derived by adding $w_{j, i}$ to all the time points in $S_{j, i}$. For example, if $S_{j, i}=\left[t^{a}, t^{b}\right]$, then $S_{j, i} \oplus w_{j, i}=\left[t^{a}+w_{j, i}, t^{b}+w_{j, i}\right] . S_{j, i}$ is initialized as $\emptyset$. We use $\tau_{j}$ to denote the minimum value of $g_{j}(t)$ for $t \in T_{j, i}-S_{j, i}$, i.e.,

$$
\tau_{j}=\min \left\{g_{j}(t) \mid t \in T_{j, i}-S_{j, i}\right\}
$$

We use $t_{j}$ to denote the minimum time point $t$ such that $g_{j}(t)$ equals to $\tau_{j}$ for $t \in T_{j, i}-S_{j, i}$, i.e.,

$$
t_{j}=\min \left\{t \mid g_{j}(t)=\tau_{j}, t \in T_{j, i}-S_{j, i}\right\}
$$

We use $R_{j, i}$ to denote time interval $\left[t_{j}, t_{a}-w_{j, i}\right]$. Then, $\tau_{j}$ is the minimum value of $g_{j}(t)$, i.e., $g_{j}\left(t^{\prime}\right)$ in Eq. (4), which minimizes $g_{j \rightarrow i}(t)$ for $t \in\left(R_{j, i}-S_{j, i}\right) \oplus w_{j, i} . g_{j \rightarrow i}(t)$ for $t \in\left(R_{j, i}-\right.$ $\left.S_{j, i}\right) \oplus w_{j, i}$ can be computed by the following equation:

$$
g_{j \rightarrow i}(t)=f_{j, i}\left(t-w_{j, i}\right)+\tau_{j}
$$

After computing $g_{j \rightarrow i}(t)$ for $t \in\left(R_{j, i}-S_{j, i}\right) \oplus w_{j, i}, S_{j, i}$ is updated as $S_{j, i} \leftarrow R_{j, i}$ and the procedure to compute $g_{j \rightarrow i}(t)$ is repeated. This procedure terminates when $S_{j, i}=T_{j, i}$. At this moment, $g_{j \rightarrow i}(t)$ is computed for any time point $t \in\left[\lambda_{j \rightarrow i}, t_{a}\right]$ because $T_{j, i} \oplus w_{j, i}=\left[\lambda_{j \rightarrow i}, t_{a}\right]$.

Note that it cannot find the minimum time point $t_{j}$ such that $g_{j}(t)=\tau_{j}$ if $g_{j}(t)=\tau_{j}$ for an open time interval, i.e., $g_{j}(t)=\tau_{j}$ for $t \in\left(t^{a}, t^{b}\right]$. In this case, $t_{j}$ is set as the left endpoint $t^{a}$ and $R_{j, i}$ is also an open time interval $\left(t_{j}, t_{a}-w_{j, i}\right]$.

We illustrate the procedure to compute $g_{j \rightarrow i}(t)$ by the example in Fig. 2. In Fig. 2, the solid line represents the cost-function $f_{j, i}(t)$ and the dash line represents the atmc-function $g_{j}(t)$. Initially, $S_{j, i}=\emptyset$ and $T_{j, i}-S_{j, i}=\left[\lambda_{j}, t_{a}-w_{j, i}\right]$. We find the minimum value of $g_{j}(t)$ is 15 for $t \in\left[\lambda_{j}, t_{a}-w_{j, i}\right]$, i.e., $\tau_{j}=15$. $t_{j}$ is the earliest time point such that $g_{j}(t)=\tau_{j}$, and thus $R_{j, i}=$ $\left[t_{j}, t_{a}-w_{j, i}\right]$. Then, we have $g_{j \rightarrow i}(t)=f_{j, i}\left(t-w_{j, i}\right)+\tau_{j}=$ $10+15=25$ for $t \in\left[t_{j}+w_{j, i}, t_{a}\right]$. Next, $S_{j, i} \leftarrow R_{j, i}$. We find the minimum value of $g_{j}(t)$ is 20 for $t \in T_{j, i}-S_{j, i}$ and $\lambda_{j}$ is the earliest time point such that $g_{j}(t)=20$. We have $R_{j, i}=\left[\lambda_{j}, t_{a}-w_{j, i}\right]$ and $R_{j, i}-S_{j, i}=\left[\lambda_{j}, t_{j}\right)$. Thus, $g_{j \rightarrow i}(t)=20+10=30$ for 
$t \in\left(R_{j, i}-S_{j, i}\right) \oplus w_{j, i}=\left[\lambda_{j \rightarrow i}, t_{j}+w_{j, i}\right)$. At this moment, $g_{j \rightarrow i}(t)$ has been computed for the whole time interval $\left[\lambda_{j \rightarrow i}, t_{a}\right]$.

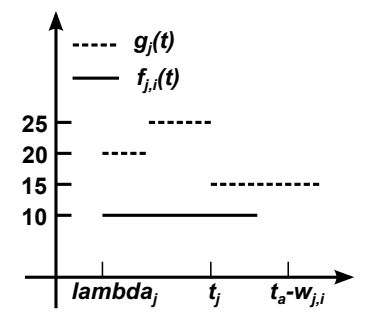

Figure 2: Computing $g_{j \rightarrow i}(t)$

The following theorem guarantees the correctness of the procedure to compute $g_{j \rightarrow i}(t)$.

Theorem 3.1: Given a time-dependent graph $G_{T}, v_{i}, v_{j} \in G_{T}$ and $v_{j} \in N^{-}\left(v_{i}\right)$. Let $g_{j}(t)$ be the atmc-function of $v_{j}$. The $g_{j \rightarrow i}(t)$ computed in the above procedure is correct, i.e., for $\forall t \in$ $\left[\lambda_{j \rightarrow i}, t_{a}\right], g_{j \rightarrow i}(t)$ is the minimum cost that one can arrive at $v_{i}$ at time point t by edge $\left(v_{j}, v_{i}\right)$ if departing from $v_{s}$ after (or at) the earliest departure time $t_{d}$.

PROOF. For $\forall t \in\left[\lambda_{j \rightarrow i}, t_{a}\right]$, we use $g_{j \rightarrow i}^{*}(t)$ to denote the minimum cost that one can arrive at $v_{i}$ via $v_{j}$ at time point $t$ by edge $\left(v_{j}, v_{i}\right)$ if departing from $v_{s}$ after (or at) time point $t_{d}$. We only need to prove $g_{j \rightarrow i}(t)=g_{j \rightarrow i}^{*}(t)$. Because $g_{j \rightarrow i}^{*}(t)$ is the minimum cost, then we have $g_{j \rightarrow i}^{*}(t) \leq g_{j \rightarrow i}(t)$. Next, we prove $g_{j \rightarrow i}(t) \leq g_{j \rightarrow i}(t)^{*}$. By the definitions of $g_{j \rightarrow i}(t)$ and $g_{j \rightarrow i}^{*}(t)$, we have the equation:

$$
g_{j \rightarrow i}^{*}(t)=g_{j}\left(t^{\prime *}\right)+f_{j, i}\left(t-w_{j, i}\right)
$$

and the equation:

$$
g_{j \rightarrow i}(t)=g_{j}\left(t^{\prime}\right)+f_{j, i}\left(t-w_{j, i}\right)
$$

where $g_{j}\left(t^{*}\right)$ and $g_{j}\left(t^{\prime}\right)$ are the costs that one arrives at $v_{j}$ for $g_{j \rightarrow i}^{*}(t)$ and $g_{j \rightarrow i}(t)$ respectively. It is obvious that $t^{\prime *} \leq t-w_{j, i}$ and $t^{\prime} \leq t-w_{j, i}$. To compute $g_{j \rightarrow i}(t), g_{j}\left(t^{\prime}\right)$ is selected as the minimum value of $g_{j}(t)$ for $t^{\prime} \leq t-w_{j, i}$. Then we have $g_{j}\left(t^{\prime}\right) \leq$ $g_{j}\left(t^{*}\right)$ and $g_{j \rightarrow i}(t) \leq g_{j \rightarrow i}^{*}(t)$.

After computing $g_{j \rightarrow i}(t)$, we can utilize $g_{j \rightarrow i}(t)$ to update $g_{i}(t)$ by the following equation:

$$
g_{i}(t)=\min \left\{g_{j \rightarrow i}(t), g_{i}(t) \mid t \in\left[\lambda_{j \rightarrow i}, t_{a}\right]\right\}
$$

In Section 3.2.1, we show that a vertex $v_{j}$ is dequeued iteratively from queue $Q$ according to $\tau_{j}$ in the first step of the TWO-STEPSEARCH algorithm. Therefore, in each iteration, we only need to compute $g_{j \rightarrow i}(t)$ and update $g_{i}(t)$ for $t \in\left(R_{j, i}-S_{j, i}\right) \oplus w_{j, i}$, for every outgoing neighbor $v_{i}$ of $v_{j}$.

\subsection{Compute the Minimum Cost}

In this section, we introduce the first step of the TwO-STEPSEARCH algorithm, i.e., how to compute the minimum cost from source $v_{s}$ to destination $v_{e}$.

\subsubsection{Main idea}

We pre-compute the earliest arrival time $\lambda_{i}$ for every vertex $v_{i} \in$ $V$. The minimum travel time to every vertex $v_{i}$ from $v_{s}$ can be computed by executing the single-source shortest path algorithm on $G_{T}$ according to the time cost $w_{i, j}$ on every edge $\left(v_{i}, v_{j}\right)$. The earliest arrival time $\lambda_{i}$ of $v_{i}$ is the sum of the earliest departure time $t_{d}$ and the minimum travel time to $v_{i}$. The time complexity of the single-source shortest path algorithm is $O(n \log n+m)$.

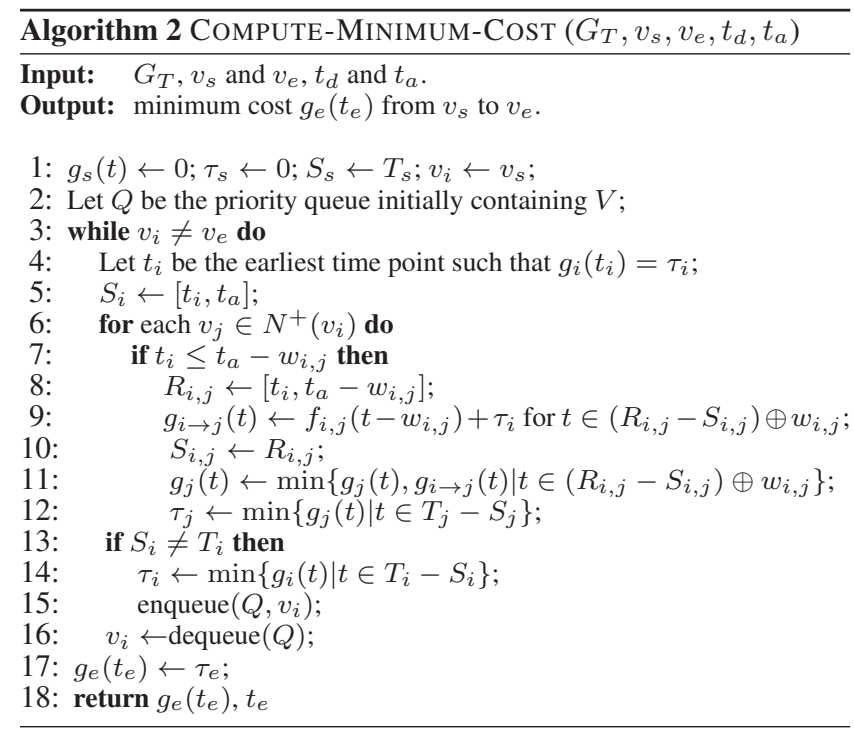

We use $g_{e}\left(t_{e}\right)$ to denote the minimum value of $g_{e}(t)$ of destination $v_{e}$, where $t_{e}$ is any time point such that $g_{e}(t)$ is minimized. Obviously, $g_{e}\left(t_{e}\right)$ is the minimum cost from $v_{s}$ to $v_{e}$ with time constraint. Our objective in this section is to compute $g_{e}\left(t_{e}\right)$.

The algorithm to compute $g_{e}\left(t_{e}\right)$ is shown in Algorithm 2. For every vertex $v_{i} \in G_{T}$, we use $T_{i}$ to denote the time interval between the earliest arrival time of $v_{i}$ and the latest arrival time $t_{a}$, i.e., $T_{i}=\left[\lambda_{i}, t_{a}\right]$. Algorithm 2 updates $g_{i}(t)$ iteratively for every vertex $v_{i} \in G_{T}$. We use $S_{i}$ to represent the processed time interval for $g_{i}(t)$ in which the minimum value of $g_{i}(t)$ has been found to update $g_{j}(t)$ for every outgoing neighbor $v_{j}$ of $v_{i}$. Different from $S_{i, j}$ discussed in Section 3.1, $S_{i}$ is updated as $\left[t_{i}, t_{a}\right]$ in every iteration, but $S_{i, j}$ is updated as $R_{i, j}=\left[t_{i}, t_{a}-w_{i, j}\right]$ for every outgoing neighbor $v_{j}$ of $v_{i}$. Let $\tau_{i}$ denote the minimum value of the current $g_{i}(t)$ for $t \in T_{i}-S_{i}$. It is obvious that $\tau_{i}$ is also the minimum value of the current $g_{i}(t)$ for $t \in T_{i, j}-S_{i, j}$ when $t_{i} \leq t_{a}-w_{i, j}$. Note that the current $g_{i}(t)$ may not be an optimal (or correct) atmc-function. Algorithm 2 updates $g_{i}(t)$ iteratively such that $g_{i}(t)$ approaches to its correct value.

For source $v_{s}, g_{s}(t), S_{s}$ and $\tau_{s}$ are initialized as $g_{s}(t) \leftarrow 0$, $S_{s} \leftarrow T_{s}$ and $\tau_{s} \leftarrow 0$ respectively. Obviously, the cost from $v_{s}$ to $v_{s}$ is zero for any arrival time $t$. It means the atmc-function $g_{s}(t)$ of $v_{s}$ equals to zero for any $t \in\left[t_{d}, t_{a}\right]$. For any other vertex $v_{i} \neq v_{s}, g_{i}(t), S_{i}$ and $\tau_{i}$ are initialized as $g_{i}(t) \leftarrow \infty, S_{i} \leftarrow \emptyset$ and $\tau_{i} \leftarrow \infty$ respectively.

Algorithm 2 utilizes a priority queue $Q$ to maintain the vertices in time-dependent graph $G_{T}$. All vertices $v_{i} \in G_{T}$ are sorted in $Q$ according to $\tau_{i}$. Algorithm 2 repeatedly dequeues the top vertex in $Q$, which has the minimum $\tau_{i}$. Initially, the top vertex in $Q$ is $v_{s}$ because $\tau_{s}=0$. The algorithm terminates when destination $v_{e}$ is dequeued from $Q$ for the first time. It means that the minimum cost from source to destination has been computed.

In every iteration, Algorithm 2 first dequeues the top vertex from $Q$, denoted as $v_{i}$. $\tau_{i}$ is the minimum value of current $g_{i}(t)$ for $t \in T_{i}-S_{i}$. Let $t_{i}$ be the earliest time point such that $g_{i}(t)=\tau_{i}$. Here, $g_{i}(t)$ is the current atmc-function of $v_{i}$. We update $S_{i}$ to $S_{i} \leftarrow\left[t_{i}, t_{a}\right]$. For each outgoing neighbor $v_{j}$ of $v_{i}$, if $t_{i} \leq t_{a}-$ $w_{i, j}$, we update $g_{j}(t)$ for $t \in\left(R_{i, j}-S_{i, j}\right) \oplus w_{i, j} . \tau_{j}$ is also updated at the same time. Note that it cannot find the earliest time point $t_{i}$ if $g_{i}(t)=\tau_{i}$ for an open interval, i.e., $g_{i}(t)=\tau_{i}$ for $t \in\left(t^{a}, t^{b}\right]$. In this case, $t_{i}$ is set as $t^{a}$ and $S_{i}$ is updated to $\left(t_{i}, t_{a}\right]$. Let $\left[t^{a}, t^{b}\right]$ 
(or $\left(t^{a}, t^{b}\right]$ ) denote the time interval such that $g_{i}(t)=\tau_{i}$, then $\tau_{i}$ is the value of the optimal (or correct) atmc-function $g_{i}(t)$ for $t \in\left[t^{a}, t^{b}\right]$. We will prove it in section 3.2.3.

After updating $g_{j}(t)$ for all the outgoing neighbors $v_{j} \in N^{+}\left(v_{i}\right)$, Algorithm 2 checks whether the processed time interval equals to the whole time interval, i.e., $S_{i}=T_{i}$. If $S_{i}=T_{i}, v_{i}$ is not necessary to update $g_{j}(t)$ for every outgoing neighbor $v_{j}$ of $v_{i}$. Then $v_{i}$ can be removed safely from $Q$. If $S_{i} \neq T_{i}$, Algorithm 2 computes the minimum value $\tau_{i}$ of current $g_{i}(t)$ for $t \in T_{i}-S_{i}$ and then enqueues $v_{i}$ back into $Q$ for further process.

When the destination $v_{e}$ is dequeued from the queue $Q$ for the first time, $\tau_{e}$ is the minimum value of $g_{e}(t)$ for $t \in\left[\lambda_{e}, t_{a}\right]$. We will prove it in Section 3.2.3. Therefore, Algorithm 2 terminates because $\tau_{e}$ is the minimum cost from the source $v_{s}$ to the destination $v_{e}$ with time constraint.

\subsubsection{Running example}

We use the example in Fig. 1 to illustrate the process to compute $g_{e}\left(t_{e}\right)$. In this example, $v_{s}=v_{1}, v_{e}=v_{4}, t_{d}=0$ and $t_{a}=60$. We first utilize the single-source shortest path algorithm to compute the earliest arrival time for all vertices $v_{1}, v_{2}, v_{3}$ and $v_{4}$ in $G_{T}$. We have $\lambda_{1}=0, \lambda_{2}=10, \lambda_{3}=15$ and $\lambda_{4}=25$. Then the time domain of $g_{1}(t), g_{2}(t), g_{3}(t)$ and $g_{4}(t)$ are $[0,60],[10,60]$, $[15,60]$ and $[25,60]$ respectively.
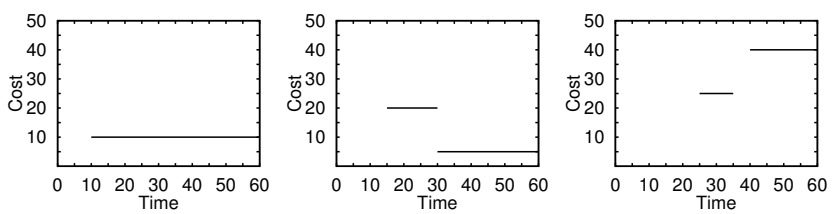

(a) $g_{2}(t)\left(1\right.$ st iteration) (b) $g_{3}(t)\left(1\right.$ st iteration) (c) $g_{4}(t)$ (2nd iteration)
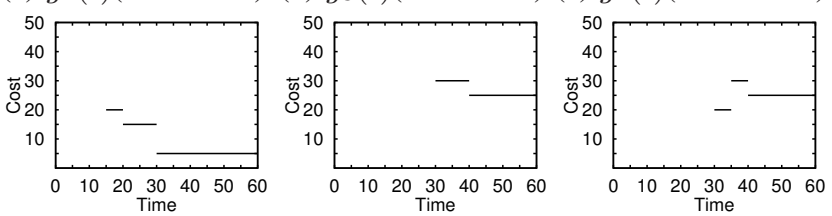

(d) $g_{3}(t)\left(3\right.$ rd iteration) (e) $g_{4}(t)\left(3\right.$ rd iteration) (f) $g_{4}(t)$ (4th iteration)

Figure 3: Running Example

Fig. 3 illustrates how to compute $g_{e}\left(t_{e}\right)$. Initially, the priority queue $Q$ contains all vertices $v_{1}, v_{2}, v_{3}$ and $v_{4}$. For source $v_{1}$, $\tau_{1}=0, S_{1}=T_{1}$ and $g_{1}(t)=0$. For other vertices $v_{i}(i=2,3,4)$, $g_{i}(t)=\infty, \tau_{i}=\infty$ and $S_{i}=\emptyset$. It implies $g_{i}(t)$ is unknown.

In the first iteration, source $v_{1}$ is dequeued from the priority $Q$, because $\tau_{1}=0$ is the minimum in $Q$. Algorithm 2 computes $g_{1 \rightarrow 2}(t)$ and $g_{1 \rightarrow 3}(t)$ and updates $g_{2}(t)$ and $g_{3}(t)$ for $v_{1}$ 's two outgoing neighbors $v_{2}$ and $v_{3}$. The resulting atmc-functions $g_{2}(t)$ and $g_{3}(t)$ are shown in Fig. 3(a) and Fig. 3(b). Because $S_{1}=T_{1}=[0,60], v_{1}$ is removed from queue $Q$ in this iteration.

In the second iteration, $v_{3}$ is the top vertex dequeued from $Q$, because $\tau_{3}=5$ is the minimum in $Q$. As shown in Fig. 3(b), $t_{3}=30$ is the earliest time such that $g_{3}(t)=\tau_{3} . S_{3}$ is updated as $S_{3} \leftarrow[30,60] . v_{4}$ is the outgoing neighbor of $v_{3}$ and $t_{3}<$ $t_{a}-w_{3,4}=50$, then Algorithm 2 computes $g_{3 \rightarrow 4}(t)$ and updates $g_{4}(t)$. The resulting $g_{4}(t)$ is shown in Fig. 3(c). Because $S_{3} \neq T_{3}$, $v_{3}$ is enqueued into $Q$ again. Here, the minimum value of current $g_{3}(t)$ for $t \in T_{3}-S_{3}=[15,30)$ is 20 , then $\tau_{3}$ is updated to 20 .

In the third iteration, the top vertex dequeued from $Q$ is $v_{2}$, because $\tau_{2}=10$ is the minimum in $Q$. As shown in Fig. 3(a), $g_{2}(t)$ equals to 10 for the whole time interval $t \in[10,60]$, then $S_{2}$ is updated as $S_{2} \leftarrow T_{2}$. For $v_{2}$ 's two outgoing neighbors $v_{3}$ and $v_{4}$, Algorithm 2 computes $g_{2 \rightarrow 3}(t)$ and $g_{2 \rightarrow 4}(t)$ and updates $g_{3}(t)$ and $g_{4}(t)$. The resulting atmc-functions $g_{3}(t)$ and $g_{4}(t)$ are shown in Fig. 3(d) and Fig. 3(e) respectively. Because $S_{2}=T_{2}, v_{2}$ is removed from $Q$ in this iteration.

In a similar way, $v_{3}$ is dequeued from $Q$ in the fourth iteration and $v_{4}$ is dequeued from $Q$ in the fifth iteration. Because $v_{4}$ is the destination, Algorithm 2 terminates. The minimum cost from source $v_{1}$ to destination $v_{4}$ is 20. As shown in Fig. 3(f), $g_{4}(t)$ equals to 20 for $t \in[30,35)$. The arrival time $t_{e}$ at $v_{4}$ can be any time point in $[30,35)$.

\subsubsection{Correctness}

Next, we prove the correctness of Algorithm 2. Let $\left[t^{a}, t^{b}\right]$ denote the time interval during which the current $g_{i}(t)$ equals to $\tau_{i}$, when $v_{i}$ is dequeued from queue $Q$. The following theorem guarantees that the value of the optimal (or correct) atmc-function $g_{i}(t)$ is $\tau_{i}$ for $t \in\left[t^{a}, t^{b}\right]$.

Theorem 3.2: Given a time-dependent graph $G_{T}$, let $v_{i}$ be the vertex dequeued from $Q$ with $\tau_{i}$ in the $k$-th iteration and $\left[t^{a}, t^{b}\right]$ be the time interval such that $g_{i}(t)=\tau_{i}$. Here, $g_{i}(t)$ is the current atmc-function of $v_{i}$. Then the value of the optimal (or correct) atmc-function $g_{i}(t)$ is $\tau_{i}$ for $t \in\left[t^{a}, t^{b}\right]$.

PROOF. For any $t^{0} \in\left[t^{a}, t^{b}\right]$, we only need to prove $g_{i}\left(t^{0}\right)=$ $\tau_{i}$, where $g_{i}(t)$ is the optimal (or correct) atmc-function of $v_{i}$. By the definition of the atmc-function, we have $g_{i}\left(t^{0}\right) \leq \tau_{i}$. Next, we need to prove $\tau_{i} \leq g_{i}\left(t^{0}\right)$. Without loss of generality, let $p$ denote the path along which one can arrive at vertex $v_{i}$ at time point $t^{0}$ with the cost $\operatorname{cost}(p)=g_{i}\left(t^{0}\right)$ :

$$
p: v_{s} \rightarrow v_{1} \rightarrow v_{2} \rightarrow \cdots \rightarrow v_{h} \rightarrow v_{i}
$$

We use $t_{x}$ to denote the arrival time at $v_{x}(1 \leq x \leq h)$ in path $p$ and $g_{x}\left(t_{x}\right)$ to denote the cost that one arrives at $v_{x}$ from $v_{s}$ along path $p$. To distinguish, we use $g_{x}^{k}(t)$ to denote the current atmcfunction of $v_{x}$ in the $k$-th iteration. It is worth noting that $g_{x}^{k}(t)$ may not be the optimal (or correct) atmc-function $g_{x}(t)$. In the $k$-th iteration, the value of $g_{h}(t)$ for $t=t_{h}$ is $g_{h}\left(t_{h}\right)$. Consider two cases: (1) $g_{h}^{k}\left(t_{h}\right)=g_{h}\left(t_{h}\right)$; and (2) $g_{h}^{k}\left(t_{h}\right)>g_{h}\left(t_{h}\right)$. Note that $g_{h}\left(t_{h}\right)$ is the value of the optimal (or correct) atmc-function of $v_{h}$ at time point $t_{h}$, then there does not exist the case of $g_{h}^{k}\left(t_{h}\right)<$ $g_{h}\left(t_{h}\right)$. In case (1), there are also two cases for $f_{h, i}\left(t^{0}-w_{h, i}\right)$ : (a) $f_{h, i}\left(t^{0}-w_{h, i}\right)=0$, and (b) $f_{h, i}\left(t^{0}-w_{h, i}\right) \neq 0$. In case (a), if $t_{h} \in S_{h}$, then $v_{h}$ has been dequeued from $Q$ with $\tau_{h} \leq$ $g_{h}^{k}\left(t_{h}\right)$. Thus $g_{i}(t)$ has been updated as $\tau_{h}+f_{h, i}\left(t-w_{h, i}\right)$. By Theorem 3.1 and $f_{h, i}\left(t^{0}-w_{h, i}\right)=0$, we have $\tau_{i} \leq \tau_{h}+f_{h, i}\left(t^{0}-\right.$ $\left.w_{h, i}\right) \leq g_{h}^{k}\left(t_{h}\right)=g_{h}\left(t_{h}\right)$. If $t_{h} \notin S_{h}$, then $\tau_{i}=g_{h}^{k}\left(t_{h}\right)=$ $g_{h}\left(t_{h}\right)$. Otherwise, $g_{h}^{k}\left(t_{h}\right)<\tau_{i}$, $v_{h}$ should be dequeued from $Q$ in the $k$-th iteration, which is in contradiction with that $v_{i}$ is dequeued from $Q$ in the $k$-th iteration. Note that $g_{h}^{k}\left(t_{h}\right)$ cannot be larger than $\tau_{i}$. Because $g_{h}^{k}\left(t_{h}\right)=g_{h}\left(t_{h}\right), g_{h}\left(t_{h}\right) \leq g_{i}\left(t^{0}\right)$ and $g_{i}\left(t^{0}\right) \leq \tau_{i}$, then $g_{h}^{k}\left(t_{h}\right) \leq \tau_{i}$. Then for case (a), we have $\tau_{i} \leq g_{h}\left(t_{h}\right)=g_{h}\left(t_{h}\right)+f_{h, i}\left(\overline{t^{0}}-w_{h, i}\right)=g_{i}\left(t^{0}\right)$. In case $(\mathrm{b})$, $f_{h, i}\left(t^{0}-w_{h, i}\right) \neq 0$, then $g_{h}^{k}\left(t_{h}\right)=g_{h}\left(t_{h}\right)<g_{i}\left(t^{0}\right)$. It indicates that $v_{h}$ has been dequeued from $Q$ with $\tau_{h} \leq g_{h}^{k}\left(t_{h}\right)$ and $g_{i}(t)$ has been updated as $\tau_{h}+f_{h, i}\left(t-w_{h, i}\right)$. By Theorem 3.1, we have

$$
\begin{aligned}
\tau_{i} & \leq \tau_{h}+f_{h, i}\left(t^{0}-w_{h, i}\right) \\
& \leq g_{h}^{k}\left(t_{h}\right)+f_{h, i}\left(t^{0}-w_{h, i}\right) \\
& =g_{h}\left(t_{h}\right)+f_{h, i}\left(t^{0}-w_{h, i}\right)=g_{i}\left(t^{0}\right)
\end{aligned}
$$

Thus for case (1), we have $\tau_{i} \leq g_{i}\left(t^{0}\right)$. Next, we prove that case (2) does not exist. Consider $v_{h-1}$. There are also two cases for 
$g_{h-1}^{k}\left(t_{h-1}\right)$ in the $k$-th iteration: $g_{h-1}^{k}\left(t_{h-1}\right)=g_{h-1}\left(t_{h-1}\right)$ and $g_{h-1}^{k}\left(t_{h-1}\right)>g_{h-1}\left(t_{h-1}\right)$. In a similar way as proving $\tau_{i}=$ $g_{i}\left(t^{0}\right)$ for case (1), we can prove $g_{h}^{k}\left(t_{h}\right)=g_{h}\left(t_{h}\right)$ if $g_{h-1}^{k}=$ $g_{h-1}\left(t_{h-1}\right)$. Then we only need to prove that there does not exist the case of $g_{h-1}^{k}\left(t_{h-1}\right)>g_{h-1}\left(t_{h-1}\right)$. Similarly, we only need to prove that there does not exist the case of $g_{h-2}^{k}\left(t_{h-2}\right)>$ $g_{h-2}\left(t_{h-2}\right)$. Recursively, we only need to prove that there does not exist the case of $g_{1}^{k}\left(t_{1}\right)>g_{1}\left(t_{1}\right)$. Because $g_{1}\left(t_{1}\right)$ has been computed when source $v_{s}$ is dequeued from $Q$ in the first iteration, then $g_{1}^{k}\left(t_{1}\right)=g_{1}\left(t_{1}\right)$. This is a contradiction. Then case (2) does not exist and we prove $\tau_{i} \leq g_{i}\left(t^{0}\right)$. Theorem 3.2 is proved.

Next, we give Theorem 3.3 to guarantee that $\tau_{i}$ is the minimum value of the optimal (or correct) atmc-function $g_{i}(t)$ for $t \in T_{i}-S_{i}$ when $v_{i}$ is dequeued from $Q$ with $\tau_{i}$.

Theorem 3.3: Given a time-dependent graph $G_{T}$, let $v_{i}$ be the vertex dequeued from $Q$ with $\tau_{i}$ in the $k$-th iteration, then $\tau_{i}$ is the minimum value of the optimal (or correct) atmc-function $g_{i}(t)$ of $v_{i}$ for $t \in T_{i}-S_{i}$.

PROOF. We prove it by contradiction and assume that there exist $t^{0} \in T_{i}-S_{i}$ such that $g_{i}\left(t^{0}\right)<\tau_{i}$. Without loss of generality, let $p$ denote the path along which one can arrive at vertex $v_{i}$ at time point $t^{0}$ with the cost $\operatorname{cost}(p)=g_{i}\left(t^{0}\right)$ :

$$
p: v_{s} \rightarrow v_{1} \rightarrow v_{2} \rightarrow \cdots \rightarrow v_{h} \rightarrow v_{i}
$$

We use $t_{x}$ to denote the arrival time at $v_{x}(1 \leq x \leq h)$ in path $p$ and $g_{x}\left(t_{x}\right)$ to denote the cost that one arrives at $v_{x}$ from $v_{s}$ along path $p$. We also use $g_{x}^{k}\left(t_{x}\right)$ to denote the current atmc-function of $v_{x}$ in the $k$-th iteration. Similar to that in Theorem 3.2, there are only two cases for $g_{i}^{k}\left(t^{0}\right)$ : (1) $g_{i}^{k}\left(t^{0}\right)=g_{i}\left(t^{0}\right)$; and (2) $g_{i}^{k}\left(t^{0}\right)>g_{i}\left(t^{0}\right)$. For case (1), $g_{i}^{k}\left(t^{0}\right)=g_{i}\left(t^{0}\right)<\tau_{i}$, then $v_{i}$ should be dequeued from $Q$ with $g_{i}^{k}\left(t^{0}\right)$ in the $k$-th iteration, which is in contradiction with that $v_{i}$ is dequeued from $Q$ with $\tau_{i}$. Then we only need to prove that case (2) does not exist. We can prove that in a similar way as proving that case (2) does not exist in Theorem 3.2. Because there is contradiction for case (1) and case (2) does not exist, then the assumption does not hold. Theorem 3.3 is proved.

Theorem 3.3 indicates that $\tau_{i}$ is the minimum value of the optimal (or correct) atmc-function $g_{i}(t)$ for $t \in T_{i}-S_{i}$ when $v_{i}$ is dequeued from $Q$. Therefore, when destination $v_{e}$ is dequeued from $Q$ for the first time, $\tau_{e}$ is the minimum value of $g_{e}(t)$ for $t \in T_{e}$, i.e., $\tau_{e}$ is the minimum cost from source $v_{s}$ to destination $v_{e}$ with time constraint.

Corollary 3.1: Given a time-dependent graph $G_{T}$, for any vertex $v_{i}$ in $G_{T}$, let $\tau_{i}^{p}$ and $\tau_{i}^{q}$ be the $\tau_{i}$ when $v_{i}$ is dequeued from $Q$ for the $p$-th and $q$-th time respectively. If $p<q$, then $\tau_{i}^{p}<\tau_{i}^{q}$.

Proof. Let $S_{i}^{p}$ and $S_{i}^{q}$ be the $S_{i}$ when $v_{i}$ is dequeued from $Q$ for the $p$-th and $q$-th time respectively. Because $p<q$, then $S_{i}^{p} \subset S_{i}^{q}$ and $T_{i}-S_{i}^{p} \supset T_{i}-S_{i}^{q}$. By Theorem $3.3, \tau_{i}^{p}$ and $\tau_{i}^{q}$ are the minimum values of the optimal (or correct) $g_{i}(t)$ for $t \in T_{i}-S_{i}^{p}$ and $t \in T_{i}-S_{i}^{q}$, then $\tau_{i}^{p}<\tau_{i}^{q}$.

\subsection{Finding the Optimal Path and the Optimal Waiting Time}

In this section, we introduce the second step of the TWO-STEPSEARCH algorithm, i.e., how to find the optimal path $p^{*}$ from source $v_{s}$ to destination $v_{e}$ and the optimal waiting time $\omega^{*}\left(v_{i}\right)$ for every vertex $v_{i} \in p^{*}$ such that $\operatorname{cost}\left(p^{*}\right)=g_{e}\left(t_{e}\right)$.

Algorithm 3 shows the algorithm to compute $p^{*}$ and $\omega^{*}\left(v_{i}\right)$ for every $v_{i} \in p^{*}$. The main idea is to find the predecessor iteratively

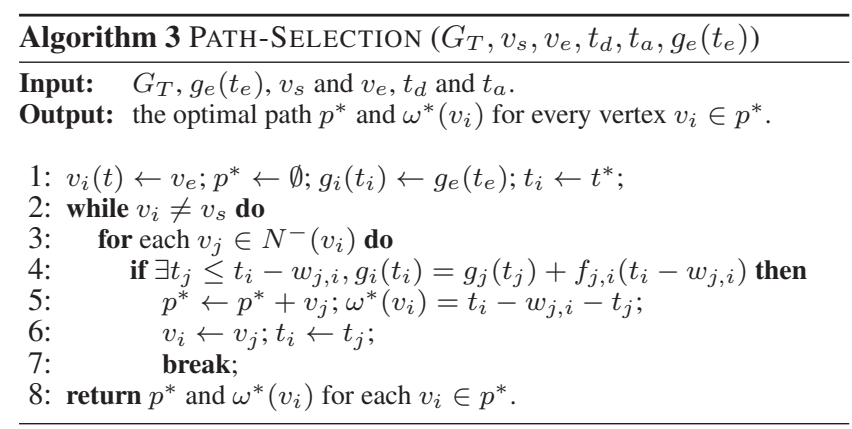

for every vertex $v_{i} \in P^{*}$ backward from destination $v_{e}$ to source $v_{s}$. Initially, $v_{i} \leftarrow v_{e}$.

In every iteration, we find the predecessor $v_{j}$ of $v_{i}$ in the optimal path $p^{*}$. Let $t_{i}$ be the arrival time at $v_{i}$ in path $p^{*}$ and $g_{i}\left(t_{i}\right)$ be the cost that one arrives at $v_{i}$ along path $p^{*}$. We initialize $g_{i}\left(t_{i}\right)$ and $t_{i}$ as $g_{e}\left(t_{e}\right)$ and $t_{e}$ respectively. For each $v_{j} \in N^{-}\left(v_{i}\right)$, if there exists a time point $t_{j}, t_{j} \leq t_{i}-w_{j, i}$, such that

$$
g_{i}\left(t_{i}\right)=g_{j}\left(t_{j}\right)+f_{j, i}\left(t_{i}-w_{j, i}\right)
$$

then $v_{j}$ is the predecessor of $v_{i}$ in path $p^{*}$ and $t_{j}$ is the arrival time at $v_{j}$ in path $p^{*}$. Such a predecessor $v_{j}$ must exist, because $g_{i}\left(t_{i}\right)$ is computed by Algorithm 2 using $g_{j}\left(t_{j}\right)$. Then the optimal waiting time at $v_{j}$ is:

$$
\omega^{*}\left(v_{j}\right)=t_{i}-w_{j, i}-t_{j}
$$

Algorithm 3 terminates when source $v_{s}$ is found as a predecessor, i.e., $v_{i}=v_{s}$. Here, all the vertices in $p^{*}$ are found and the optimal waiting time $\omega^{*}\left(v_{i}\right)$ for every vertex $v_{i} \in p^{*}$ is computed.

We use the example in Fig. 3 to illustrate the process to compute $p^{*}$ and $\omega^{*}\left(v_{i}\right)$ for every vertex $v_{i} \in p^{*}$. From Fig. 3, we find that the minimum cost from $v_{1}$ to $v_{4}$ is $g_{4}\left(t_{4}\right)=20$. Here, $g_{4}(t)=20$ for $t \in[30,35)$, then $t_{4}$ can be any time point in $[30,35)$, e.g., $t_{4}=30$. For a $v_{4}$ 's incoming neighbor $v_{3}$, the departure time from $v_{3}$ is $t_{4}-w_{3,4}=30-10=20$. We find that $g_{3}\left(t_{3}\right)=15$ when $t_{3}=20$ and then we have:

$g_{3}\left(t_{3}\right)+f_{3,4}\left(t_{4}-w_{3,4}\right)=g_{3}(20)+f_{3,4}(30-10)=20=g_{4}\left(t_{4}\right)$

Thus $v_{3}$ is the predecessor of $v_{4}$ in the optimal path $p^{*}$. The optimal waiting time at $v_{3}$ is $\omega^{*}\left(v_{3}\right)=t_{4}-w_{3,4}-t_{3}=0$.

In the second iteration, for a $v_{3}$ 's incoming neighbor $v_{2}$, the departure time from $v_{2}$ is $t_{3}-w_{2,3}=20-5=15 . g_{2}\left(t_{2}\right)=10$ when $t_{2}=10$ and then we have:

$$
g_{2}\left(t_{2}\right)+f_{2,3}\left(t_{3}-w_{2,3}\right)=10+5=15=g_{3}\left(t_{3}\right)
$$

Thus $v_{2}$ is the predecessor of $v_{3}$ in the optimal path $p^{*}$ and the optimal waiting time at $v_{2}$ is $\omega^{*}\left(v_{2}\right)=t_{3}-w_{2,3}-t_{2}=5$.

In the similar way, $v_{1}$ is found as the predecessor of $v_{2}$ in path $p^{*}$. Because $v_{1}$ is the source vertex, Algorithm 3 terminates. Then the optimal path $p^{*}$ is $v_{1} \rightarrow v_{2} \rightarrow v_{3} \rightarrow v_{4}$. The optimal waiting time for all the vertices in the optimal path $p^{*}$ are $\omega^{*}\left(v_{1}\right)=0$, $\omega^{*}\left(v_{2}\right)=5$ and $\omega^{*}\left(v_{3}\right)=0$ respectively.

\subsection{Time and Space Complexities}

We first analyze the time complexities of Algorithm 2 and Algorithm 3 and then give the time complexity of the TWO-STEPSEARCH algorithm (Algorithm 1). Let $n$ and $m$ be the number of vertices and edges in $G_{T}$ respectively, and $k$ be the average number of piecewise constant values of atmc-function $g_{i}(t)$ in $G_{T}$.

Lemma 3.1: The time complexity of COMPUTE-Minimum-COST (Algorithm 2) is $O(k n \log n+m k)$. 
PROOF. In every iteration of Algorithm 2, there are at most $n$ vertices maintained in $Q$ at the same time. Using Fibonacci Heap [6], dequeue $(Q)$ and enqueue $(Q)$ require $O(\log n)$ and $O(1)$ amortized time respectively. When a vertex $v_{i}$ is dequeued from $Q$, Algorithm 2 needs to compute $g_{i \rightarrow j}(t)$ and update $g_{j}(t)$ for every outgoing neighbor $v_{j}$ of $v_{i}$. To compute $g_{i \rightarrow j}(t)$, Algorithm 2 needs to add $\tau_{i}$ to every constant value of $f_{i, j}(t)$ for $t \in R_{i, j}-S_{i, j}$. We use $k_{i, j}\left[I^{p}\right]$ to denote the number of piecewise constant values of $f_{i, j}(t)$ for $t \in R_{i, j}-S_{i, j}$ when $v_{i}$ is dequeued from $Q$ for the $p$-th time. Here $I^{p}=R_{i, j}-S_{i, j}$. Then the time complexity is $O\left(k_{i, j}\left[I^{p}\right]\right)$ for computing $g_{i \rightarrow j}(t)$ and updating $g_{j}(t)$. When $v_{i}$ is dequeued from $Q$ for the $p$-th time, the time complexity for this iteration is $O\left(\sum_{x=1}^{d^{+}\left(v_{i}\right)} k_{i, j_{x}}\left[I^{p}\right]+\log n\right)$, where $v_{j_{x}}$ is the $x$-th outgoing neighbor $v_{j}$ of $v_{i}$. We call $t_{i}^{p}$ as an "connection point" of $I_{p}$ and $I_{p+1}$, where $t_{i}^{p}$ is $t_{i}$ when $v_{i}$ is dequeued from $Q$ for the $p$-th time. For a constant value $c_{x}$ of $f_{i, j}(t)$, assume $c_{x}$ 's corresponding time interval is $\left[t^{x-1}, t^{x}\right]$, i.e., $f_{i, j}(t)=c_{x}$ for $t \in\left[t^{x-1}, t^{x}\right]$. Note that $c_{x}$ is counted in both $k\left[I^{p}\right]$ and $k\left[I^{p+1}\right]$ if the connection point $t_{i}^{p} \in\left(t^{x-1}, t^{x}\right)$. Here, $c_{x}$ is the first constant value of $f_{i, j}(t)$ for $t \in I^{p}$ and the last one of $f_{i, j}(t)$ for $t \in I^{p+1}$. Let $l_{i}$ be the number of times that $v_{i}$ is dequeued from $Q$ in total. There are at most $\left(l_{i}-1\right)$ connection points, then $\sum_{p=1}^{l_{i}} k_{i, j}\left[I^{p}\right] \leq k_{i, j}\left[T_{i}\right]+\left(l_{i}-1\right)$. Thus for $v_{i}$, the time complexity is:

$$
\begin{aligned}
& O\left(\sum_{p=1}^{l_{i}}\left(\sum_{x=1}^{d^{+}\left(v_{i}\right)} k_{i, j_{x}}\left[I^{p}\right]+\log n\right)\right) \\
\leq & O\left(\sum_{x=1}^{d^{+}\left(v_{i}\right)}\left(k_{i, j_{x}}\left[T_{i}\right]+\left(l_{i}-1\right)\right)+\sum_{p=1}^{l_{i}} \log n\right)
\end{aligned}
$$

and the total time complexity is:

$$
\begin{aligned}
& O\left(\sum_{v_{i} \in V}\left(\sum_{x=1}^{d^{+}\left(v_{i}\right)}\left(k_{i, j_{x}}\left[T_{i}\right]+\left(l_{i}-1\right)\right)+\sum_{p=1}^{l_{i}} \log n\right)\right) \\
= & O\left(\sum_{v_{i} \in V} \sum_{x=1}^{d^{+}\left(v_{i}\right)}\left(k_{i, j_{x}}\left[T_{i}\right]+\left(l_{i}-1\right)\right)+\sum_{v_{i} \in V} \sum_{p=1}^{l_{i}} \log n\right)
\end{aligned}
$$

It is obvious that $\sum_{v_{i} \in V} \sum_{x=1}^{d^{+}\left(v_{i}\right)} k_{i, j_{x}}\left[T_{i}\right]$ equals to the total number of constant values of all the cost functions on all the edges in $G_{t}$. Let $k^{\prime}$ be the average number of constant values of cost function $f_{i, j}(t)$ in $G_{T}$, then $m k^{\prime}=\sum_{v_{i} \in V} \sum_{x=1}^{d^{+}\left(v_{i}\right)} k_{i, j_{x}}\left[T_{i}\right]$. Let $k_{i}$ denote the number of constant values of atmc-function $g_{i}(t)$. By Theorem 3.3 and Corollary 3.1, we know that $v_{i}$ is dequeued from $Q$ repeatedly according to the different constant values (i.e., $\tau_{i}$ ) of $g_{i}(t)$ in Algorithm 2. Thus $v_{i}$ is dequeued from $Q$ at most $k_{i}$ times, then $l_{i} \leq k_{i}$ and $\sum_{v_{i} \in V} \sum_{x=1}^{d^{+}\left(v_{i}\right)}\left(l_{i}-1\right)<\sum_{x=1}^{d^{+}\left(v_{i}\right)} \sum_{v_{i} \in V} k_{i}$. Here, $\sum_{v_{i} \in V} k_{i}$ equals to the total number of constant values of all the atmc-functions in $G_{T}$. Thus we have $\sum_{v_{i} \in V} k_{i}=n k$ and $\sum_{x=1}^{d^{+}\left(v_{i}\right)} \sum_{v_{i} \in V} k_{i}=m k$. Similarly, $\sum_{v_{i} \in V} \sum_{p=1}^{l_{i}} \log n=$ $n k \log n$. Then the total complexity is $O\left(m\left(k^{\prime}+k\right)+n k \log n\right)$. Because every $g_{i}(t)$ are computed by different $f_{i, j}(t)$, then $k^{\prime} \leq k$. Thus the total time complexity is $O(n k \log n+m k)$.

Lemma 3.2: The time complexity of PATH-SELECTION (Algorithm 3) is $O(m k)$.

PROOF. In every iteration of Algorithm 3 , for a vertex $v_{i}$ in the optimal path $p^{*}$, all $v_{i}$ 's incoming neighbors need to be examined. For every incoming neighbor $v_{j}$ of $v_{i}$, Algorithm 3 needs to check whether there exists a time $t_{j}\left(t_{j} \leq t_{i}-w_{j, i}\right)$, such that Eq. (6) holds. This operation requires $O(k)$ time. Thus the time complexity of computing predecessor for $v_{i}$ is $d^{-}\left(v_{i}\right) k$ in every iteration. Because there is no loop in the optimal path $p^{*}$, then Algorithm 3 needs to compute predecessor for $v_{i}$ at most once. Thus, the time complexity of Algorithm 1 is $O\left(\sum_{v_{i} \in V}\left(d^{-}\left(v_{i}\right) k\right)\right)=O(m k)$. $\square$

By Lemma 3.1 and Lemma 3.2, then we have the time complexity of Algorithm 2.

Theorem 3.4: The time complexity of TWO-STEP-SEARCH (Algorithm 1) is $O(k n \log n+m k)$.

Proof. Algorithm 2 and Algorithm 3 are two steps of Algorithm 1. By Lemma 3.1 and Lemma 3.2, the time complexity of Algorithm 1 (TwO-STEP-SEARCH) is $O(k n \log n+m k)$.

Next,we analyze the space complexity of Algorithm 1.

Theorem 3.5: The space complexity of TWO-STEP-SEARCH (Algorithm 1) is $O((n+m) k)$.

PROOF. Algorithm 2 and Algorithm 3 are two steps of Algorith$\mathrm{m} 1$. Algorithm 2 needs to maintain at most $n$ vertices in $Q$. For every vertex $v_{i}$, Algorithm 2 and Algorithm 3 need to maintain its atmc-function $g_{i}(t)$. For every edge $\left(v_{i}, v_{j}\right)$ in $G_{T}$, Algorithm 2 and Algorithm 3 need to maintain its cost function $f_{i, j}(t)$. Therefore, the space complexity of Algorithm 1 is $O((n+m) k)$.

\subsection{Discussion about Time Function}

In the above discussion, we assume that travel time for every edge $\left(v_{i}, v_{j}\right)$ is a constant value $w_{i, j}$. Next, we discuss how to handle the case where travel time is a function $w_{i, j}(t)$ which is related to the departure time $t$ from $v_{i}$. In this paper, we assume that $G_{T}$ has the FIFO property. FIFO property for an edge $\left(v_{i}, v_{j}\right)$ implies that if departing earlier from $v_{i}$, one arrives earlier at $v_{j}$.

Definition 3.1: (FIFO) Given a time-dependent graph $G_{T}$, we say $G_{T}$ is a FIFO graph if and only if the time function $w_{i, j}(t)$ of every edge $\left(v_{i}, v_{j}\right)$ has the FIFO property, i.e., $w_{i, j}\left(t_{0}\right)<\Delta t+w_{i, j}\left(t_{0}+\right.$ $\Delta t)$ for $\Delta t>0$, or $t_{1}+w_{i, j}\left(t_{1}\right)<t_{2}+w_{i, j}\left(t_{2}\right)$ for $t_{1}<t_{2} . \quad \square$

The restriction of the FIFO property is reasonable and many previous works also make this assumption $[16,13]$. Consider a road network, for two cars towards the same road segment, the first one reaching the starting point should leave the end point first.

We use arrive $_{i, j}(t)$ to denote the arrival-time function of edge $\left(v_{i}, v_{j}\right)$, arrive $e_{i, j}(t)=t+w_{i, j}(t)$. arrive $i, j(t)$ indicates the arrival time at $v_{j}$ if one departs from $v_{i}$ at time point $t$. It is obvious that $\operatorname{arrive}_{i, j}(t)$ is a one-one mapping function. Given an arrival time $t$ at $v_{j}$, we can compute the departure time from $v_{i}$ by arrive $e_{i, j}^{-1}(t)$, where $\operatorname{arrive}_{i, j}^{-1}(t)$ is the inverse function of $\operatorname{arrive}_{i, j}(t)$.

As similar as Eq. (4) in Section 3.1, for every edge $\left(v_{j}, v_{i}\right)$, given the arrival time $t$ for $v_{i}$, the departure time from $v_{j}$ is fixed as arrive $_{j, i}^{-1}(t)$ and the cost to travel edge $\left(v_{j}, v_{i}\right)$ is $f_{j, i}\left(\operatorname{arrive}_{j, i}^{-1}(t)\right)$. Therefore, $g_{j \rightarrow i}(t)$ can be computed by the following equation:

$$
g_{j \rightarrow i}(t)=\min _{t^{\prime} \leq \operatorname{arrive}_{j, i}^{-1}(t)}\left\{g_{j}\left(t^{\prime}\right)\right\}+f_{j, i}\left(\operatorname{arrive}_{j, i}^{-1}(t)\right)
$$

There is a special case where $w_{j, i}(t)$ is a non-continuous function. For example, $w_{j, i}(t)$ is a function $w_{j, i}^{1}(t)$ for $t \leq t^{\dagger}$ and another function $w_{j, i}^{2}(t)$ for $t>t^{\dagger}$. Here $w_{j, i}^{1}(t)$ is always smaller than $w_{j, i}^{2}(t)$. Obviously, $w_{j, i}(t)$ is broken at $t^{\dagger}$. Then the arrivaltime function for edge $\left(v_{j}, v_{i}\right)$ is non-continuous, because one cannot arrive at $v_{i}$ between time $t^{\dagger}+w_{j, i}^{1}\left(t^{\dagger}\right)$ and $t^{\dagger}+w_{j, i}^{2}\left(t^{\dagger}\right)$. We call $t^{\dagger}$ as a "break point" of $w_{j, i}(t)$. Suppose there exists a "break point" $t^{\dagger}$ in time interval $\left[t^{a}, t^{b}\right]$ during which $f_{j, i}(t)=c$, then $g_{j \rightarrow i}(t)$ equals to $c+\tau_{j}$ for $t \in\left[\operatorname{arrive}_{j, i}^{1}\left(t^{a}\right)\right.$, $\left.\operatorname{arrive}_{j, i}^{1}\left(t^{\dagger}\right)\right]$ and 


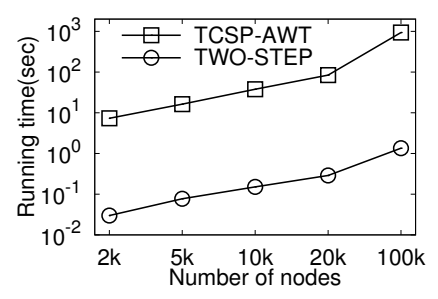

(a) querying time

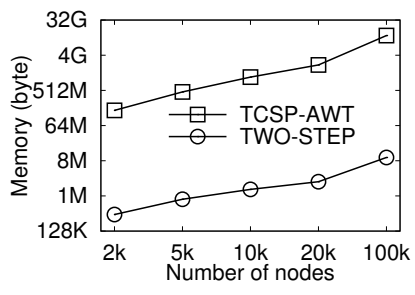

(b) memory overhead

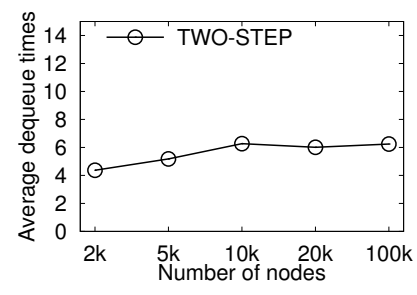

(c) average dequeue times

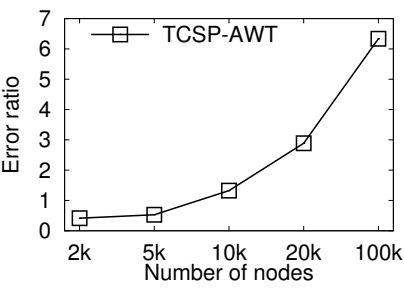

(d) relative error

Figure 4: Impact of vertex size

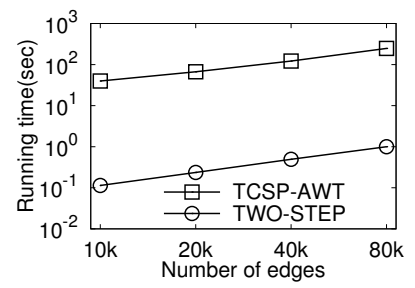

(a) querying time

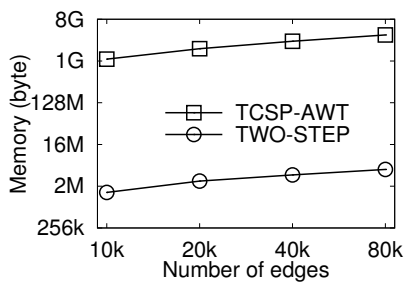

(b) memory overhead

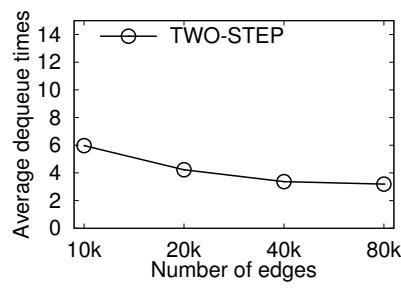

(c) average dequeue times

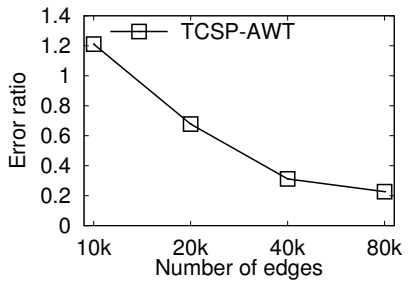

(d) relative error

Figure 5: Impact of edge size

$t \in\left(\operatorname{arrive}_{j, i}^{2}\left(t^{\dagger}\right), \operatorname{arrive}_{j, i}^{2}\left(t^{b}\right)\right]$, respectively. Here arrive ${ }_{j, i}^{1}(t)=$ $t+w_{j, i}^{1}(t)$ and arrive ${ }_{j, i}^{2}(t)=t+w_{j, i}^{2}(t)$. In addition, $g_{j \rightarrow i}(t)=\infty$ for $t \in\left(\operatorname{arrive}_{j, i}^{1}\left(t^{\dagger}\right)\right.$, $\left.\operatorname{arrive}_{j, i}^{2}\left(t^{\dagger}\right)\right]$. Note that $\operatorname{arrive}_{j, i}^{1}\left(t^{\dagger}\right)<$ $\operatorname{arrive}_{j, i}^{2}\left(t^{\dagger}\right)$. It indicates one cannot arrive at $v_{i}$ by edge $\left(v_{j}, v_{i}\right)$ between time arrive ${ }_{j, i}^{1}\left(t^{\dagger}\right)$ and $\operatorname{arrive}_{j, i}^{2}\left(t^{\dagger}\right)$.

As similar as Eq. (6), Algorithm 3 can compute the predecessor $v_{j}$ for a vertex $v_{i}$ in the optimal path $p^{*}$ by the following equation if travel time is a function $w_{i, j}(t)$ for every edge $\left(v_{i}, v_{j}\right) \in G_{T}$.

$$
g_{i}\left(t_{i}\right)=g_{j}\left(t_{j}\right)+f_{j, i}\left(\operatorname{arrive}_{j, i}^{-1}\left(t_{i}\right)\right)
$$

The optimal waiting time for $v_{j}$ can be computed as follows:

$$
\omega^{*}\left(v_{j}\right)=t_{i}-w_{j, i}\left(\operatorname{arrive}_{j, i}^{-1}\left(t_{i}\right)\right)-t_{j}
$$

\section{PERFORMANCE EVALUATION}

We compare the TWO-STEP-SEARCH algorithm with the TCSPAwT (Time Constraint Shortest Path Allow Waiting Time) algorithm [2] based on two real datasets. TCSP-AWT is the state of the art algorithm to compute the cost-optimal path with time constraint in time-dependent graphs. However, TCSP-AWT can be only used for the discrete time model. All the experiments are conducted on a $3.0 \mathrm{GHz}$ Intel Core i5 CPU PC with the $32 \mathrm{~GB}$ main memory, running on Windows 7.

\subsection{Dataset and Experiment Setup}

We employ the following two real road networks:

CARN: This dataset is California road network including 23,718 vertices and 33,561 edges. A vertex represents an intersection or a road endpoint and an edge represents a road segment.

EURN: This network describes Eastern USA road network and it includes 3,598,623 vertices and 8,778,114 edges. As the same as the CARN, a vertex represents an intersection or a road endpoint and an edge represents a road segment.

We generate four time-dependent graphs with different sizes using the CARN dataset and five time-dependent graphs with different sizes using the EURN datasets. For graphs of CARN dataset, the number of vertices ranges from $2 \mathrm{k}$ to $20 \mathrm{k}$. For graphs of EU$\mathrm{RN}$ dataset, the number of vertices ranges from one million to three millions. We generate travel time according to the road length. The travel time for an edge $\left(v_{i}, v_{j}\right)$ is more if the road represented by $\left(v_{i}, v_{j}\right)$ is longer. To simulate the real traffic case, we compute the betweenness centrality for every edge in $G_{t}$ and sort all the edges in descending order of betweenness. We select the top $20 \%$ edges as the traffic hubs in a road network. We assign the more expensive travel cost and the more travel time on these edges. The time domain is set as $\mathcal{T}=[0,2000]$, i.e., the departure time $t$ can be selected from $[0,2000]$ for any vertex in a graph. Here, 2000 means 2000 time units. For every $f_{i, j}(t)$, we split the time domain $\mathcal{T}$ to $k$ subintervals and assign a constant value randomly for every subinterval and then it is a piecewise constant function. For every $w_{i, j}(t)$, the time domain $T$ is also randomly divided to $k$ subintervals $\left(\left[t_{0}, t_{1}\right],\left[t_{1}, t_{2}\right], \cdots,\left[t_{k-1}, t_{k}\right]\right)$, where $t_{0}$ and $t_{k}$ are the start and the end of the time domain $T$ respectively. The value of $w_{i, j}\left(t_{0}\right)$ is first generated as a random number from $[0, \bar{w}]$, where $\bar{w}$ is a number to restrict the max value of $w_{i, j}(t)$. Within each subinterval $\left[t_{x-1}, t_{x}\right](1 \leq x \leq k), w_{i, j}(t)$ is a linear function $w_{i, j}^{x}(t), w_{i, j}^{x}\left(t_{x-1}\right)=w_{i, j}^{x-1}\left(t_{x-1}\right)$ and $w_{i, j}^{x}\left(t_{x}\right)$ is generated as a random number from $\left[\max \left(0, w_{i, j}^{x}\left(t_{x-1}\right)-\Delta t_{x}\right), \bar{w}\right]$, where $\Delta t_{x}=t_{x}-t_{x-1}$. Then the time function $w_{i, j}(t)$ is guaranteed to be non-negative and FIFO. For TCSP-AWT, we sample one discrete time point every other time unit from the whole time interval. For example, if the whole time interval is $[0,1000]$, then the sampled discrete time points are $[1,3,5, \cdots, 999]$. We randomly generate 1,000 pairs of vertices and query the cost-optimal path between every pair of vertices. The reported querying time is the average time on each dataset. We use TwO-STEP to denote TwO-STEPSEARCH in the experimental results.

We are interested in the following aspects to evaluate the performance of TWO-STEP-SEARCH: (1) the impact of number of vertices; (2) the impact of number of edges; (3) the impact of distances between source and destination; (4) the impact of the length of time interval $\left[t_{d}, t_{a}\right]$ between the earliest departure time $t_{d}$ and the latest arrival time $t_{a}$; and (5) the impact of the average number of piecewise intervals of $f_{i, j}(t)$. The parameters to be evaluated are: (1) querying time; (2) memory overhead; (3) average number of times that a vertex is dequeued from $Q ;(4)$ relative error $\left(c-c^{*}\right) / c^{*}$ of TCSP-AWT. Note that TCSP-AWT can be only used for the discrete 


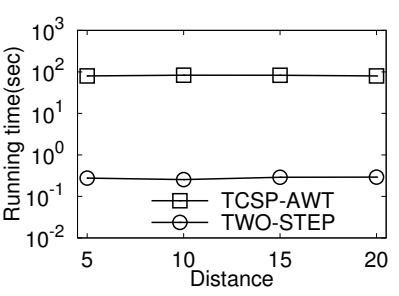

(a) querying time

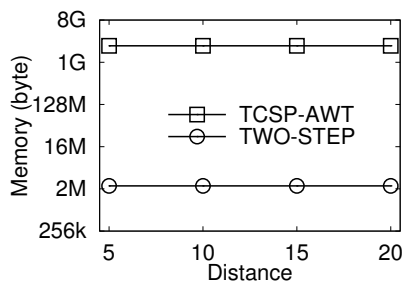

(b) memory overhead

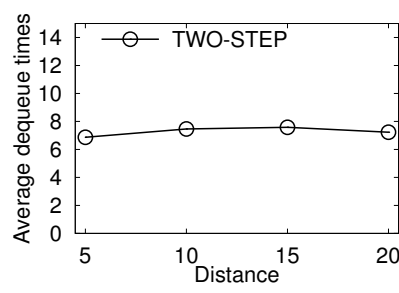

(c) average dequeue times

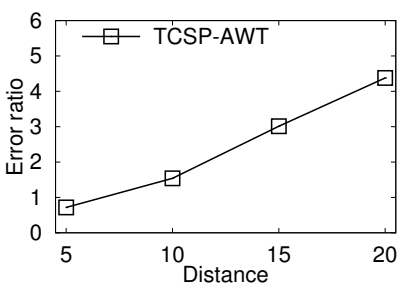

(d) relative error

Figure 6: Impact of distance between source and destination

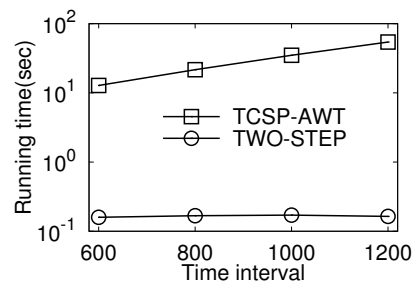

(a) querying time

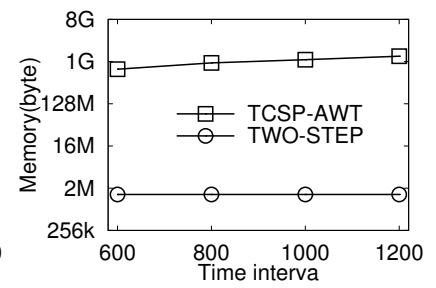

(b) memory overhead

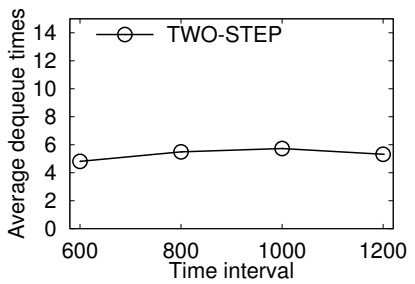

(c) average dequeue times

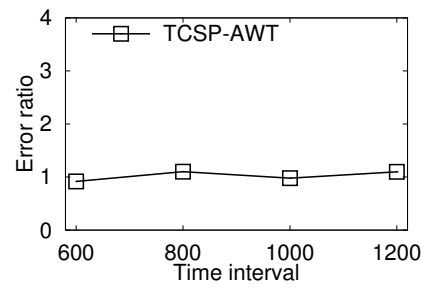

(d) relative error

Figure 7: Impact of the length of time interval $\left[t_{d}, t_{a}\right]$

time model, thus the results of TCSP-AWT may not be optimal as the discussion in Section 2.2. Here, $c$ is the cost of the path computed by TCSP-AWT and $c^{*}$ is the cost of the optimal path. There is no error for TWO-STEP-SEARCH algorithm.

\subsection{Experimental Results}

Exp-1. Impact of the number of vertices: In Fig. 4, we study the impact of number of vertices of time dependent graph $G_{T}$. In this group of the experiments, the number of vertices increases from $2 \mathrm{~K}$ to $100 \mathrm{~K}$, where the graphs with $2 \mathrm{k}$ to $20 \mathrm{k}$ vertices are generated from CARN dataset and the graph with $100 \mathrm{k}$ vertices is generate from EURN dataset. The number of piecewise intervals of $f_{i, j}(t)$ is 10 . The time interval $\left[t_{d}, t_{a}\right]$ is $[0,1000]$. As shown in Fig. 4(a) and Fig. 4(b), the querying time and memory overhead of TwO-STEP-SEARCH are always less than that of TCSP-AWT. TWO-STEP-SEARCH is nearly 20 times faster than TCSP-AWT. The memory overhead of TWO-STEP-SEARCH is nearly 500 times less than that of TCSP-AWT. The reason is TCSP-AWT needs to compute the arrival costs for all the vertices in $G_{T}$ for every time point and then it can compute the minimum cost from source to destination based on these results. We find the querying time and memory overhead increase marginally with the number of vertices increasing. From Fig. 4(c), we find the average number of dequeuing times of vertices is not affected by the number of vertices. From Fig. 4(d), we find the relative error increases with the increasing of the number of vertices. If the number of vertices increases, the distance between source and destination increases. Thus the relative error cumulated in the path from source to destination increases.

Exp-2. Impact of the number of edges: We study the impact of the number of edges in Fig. 5. In this group of experiments, the number of vertices is fixed at $10 \mathrm{~K}$ and the number of edges increases from $10 \mathrm{~K}$ to $80 \mathrm{~K}$. The number of piecewise intervals of $f_{i, j}(t)$ is 10 . The time interval $\left[t_{d}, t_{a}\right]=[0,1000]$. As shown in Fig. 5(a) and Fig. 5(b), we find TwO-STEP-SEARCH always performs better than TCSP-AWT. From Fig. 5(c), we find the average number of dequeuing times decreases with the increasing of the number of edges. Intuitively, more edges results in larger density of graphs and then the distance between every two vertices decreases. Thus
TWO-STEP-SEARCH can compute the minimum cost from source to destination faster and the average number of dequeuing times decreases. However, more edges indicates more outgoing neighbors of a vertex in a graph. When a vertex $v_{i}$ is dequeued from $Q$, it takes more time to update the atmc-functions for all the outgoing neighbors of $v_{i}$. Therefore, in Fig. 5(a), the querying time increases with the number of edges increasing. From Fig. 5(d), we find the relative error of TCSP-AWT decreases with the number of edges increasing. It is because the increasing of the number of edges shortens the distance between source and destination. Then less relative error is cumulated in the path from source to destination.

Exp-3. Impact of the distance between source and destination: In Fig. 6, we study the impact of the distance between source and destination. In this group of experiments, the number of vertices is fixed at $20 \mathrm{~K}$. The distance between source and destination ranges from 5 to 20. As shown in Fig. 6(a) and Fig. 6(b), we find the querying time and memory overhead of both algorithms are not affected by the distance between source and destination. The reason is the path with the minimum distance is not a cost-optimal path in many cases. Note that the density of a graph does not increase in this group of experiments. Thus, in Fig. 6(c), the average number of dequeuing times is not affected by the distance. From Fig. 6(d), we find the relative error of TCSP-AWT increases with the increasing of distance. The longer distance between source and destination, the more error cumulated in the path from source to destination.

Exp-4. Impact of the length of time interval $\left[t_{d}, t_{a}\right]$ : In Fig. 7, we study the impact of the length of time interval $\left[t_{d}, t_{a}\right]$. In this group of experiments, the earliest departure time is fixed at time point 0 and the latest arrival time ranges from time point 600 to time point 1200. As shown in Fig. 7(a) and Fig. 7(b), the querying time and memory overhead of TWO-STEP-SEARCH are not affected by the length of time interval $\left[t_{d}, t_{a}\right]$. It is because the number of piecewise intervals of $f_{i, j}(t)$ does not increase. However, the number of sampled discrete time points for TCSP-AWT increases with the length of time interval $\left[t_{d}, t_{a}\right]$ increasing. Thus the querying time and memory overhead of TCSP-AWT increases. We also find the average number of dequeuing times is stable in Fig. 7(c) and the relative error does not change significantly in Fig. 7(d). 


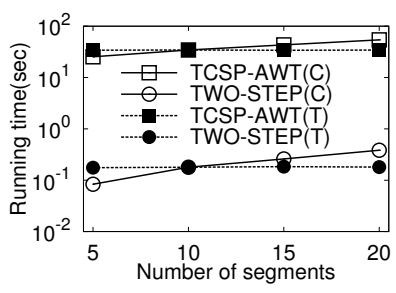

(a) querying time

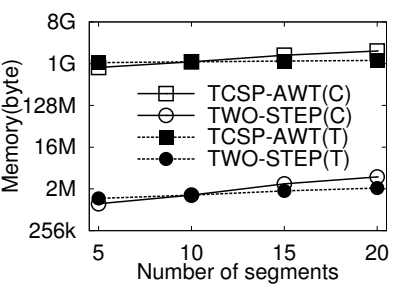

(b) memory overhead

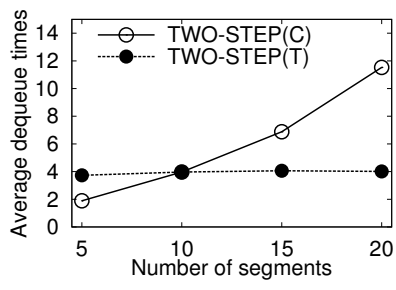

(c) average dequeue times

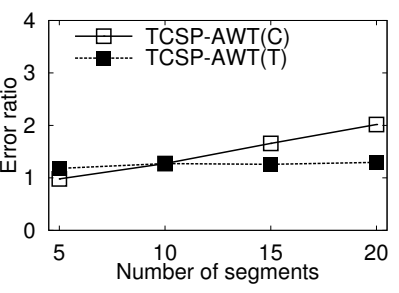

(d) relative error

Figure 8: Impact of number of piecewise intervals of cost-function and time-function

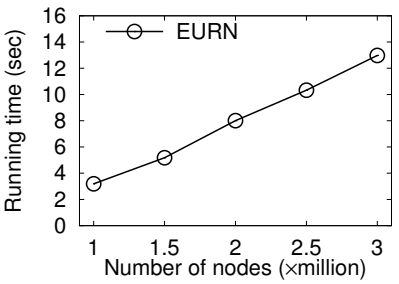

(a) querying time (nodes)

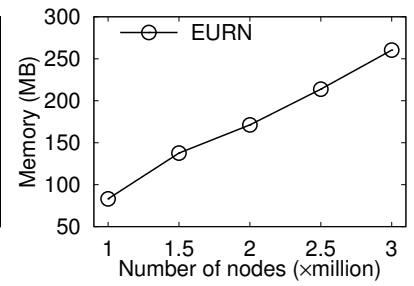

(b) memory overhead (nodes)

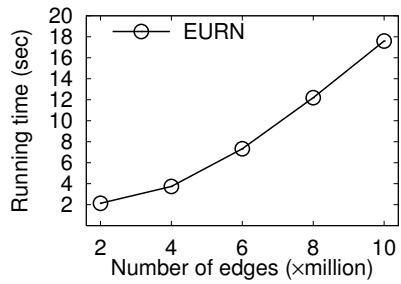

(c) querying time (edges)

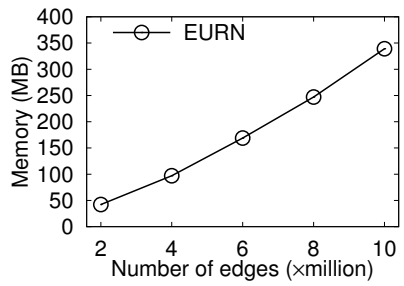

(d) memory overhead (edges)

Figure 9: Adaptivity to large graphs

Exp-5. Impact of the number of piecewise intervals of cost function and time function: In Fig. 8, we investigate the impact of the number of piecewise intervals of $f_{i, j}(t)$ by curves TCSPAWT $(C)$ and TWO-STEP $(C)$ in Fig. 8. In this group of experiments, the number of piecewise intervals of $f_{i, j}(t)$ increases from 5 to 20. As shown in Fig. 8(a) and Fig. 8(b), the querying time and memory overhead of TWO-STEP-SEARCH increase with the increasing of the number of piecewise intervals. The reason is that TWO-STEP-SEARCH needs more time to update the atmc-function for vertices when the number of piecewise time intervals increases. We find TwO-STEP-SEARCH is always better than TCSP-AWT. From Fig. 8(c), we find the average number of dequeuing times increases with the increasing of the number of piecewise intervals. In Fig. 8(d), the relative error of TCSP-AWT increases with the increasing of the number of piecewise intervals. When the number of piecewise intervals increases, the number of arrival cost values between two consecutive discrete time points in TCSP-AWT increases. This results in that TCSP-AWT cannot find the optimal cost of traveling an edge. Thus the relative error of TCSP-AWT increases with the number of piecewise intervals increasing.

We also investigate the impact of the number of piecewise intervals of $w_{i, j}(t)$ by curves TCSP-AWT(T) and TWO-STEP(T) in Fig. 8. The number of piecewise intervals of $w_{i, j}(t)$ also increases from 5 to 20. The experimental results show the efficiency of TWO-STEP-SEARCH is not affected by the number increasing. It is because the increasing of this number does not incur extra operation on atmc-function during the whole process.

Exp-6. Scalability: We evaluate the scalability of TwO-STEPSEARCH in Fig.9. We investigate the querying time and memory overhead by varying the number of vertices from one million to three millions and by varying the number of edges from two millions to ten millions on the EURN dataset. In this group of experiments, the number of piecewise intervals of $f_{i, j}(t)$ and $w_{i, j}(t)$ are both 10 . The time interval $\left[t_{d}, t_{a}\right]$ is $[0,1000]$. The experimental results show TWO-STEP-SEARCH can perform efficiently even though the number of vertices is larger than three millions or the number of edges is larger than ten millions. These experimental results indicates TWO-STEP-SEARCH are also suitable for large time-dependent graphs.

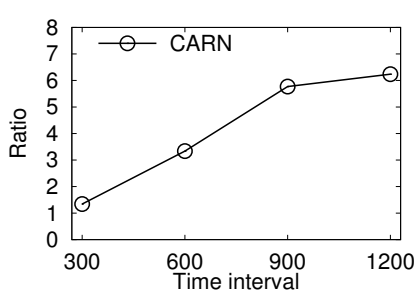

(a) varying time interval

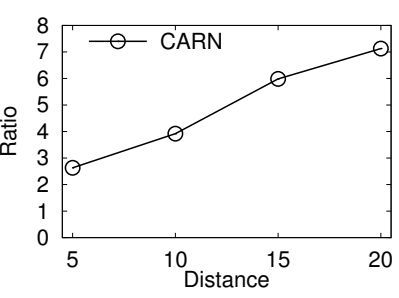

(b) varying distance
Figure 10: The cost-optimal path vs the fastest path

Exp-7. Comparing with the fastest path: In Fig. 10, we investigate the ratio $\frac{c^{\prime}}{c *}$ on the CARN dataset with $20 \mathrm{~K}$ vertices, where $c^{\prime}$ is the travel cost of the path with the minimum travel time from $v_{s}$ to $v_{e}$ and $c *$ is the cost of the path computed by TWO-STEPSEARCH, i.e., the cost-optimal path with time constraint. $\frac{c^{\prime}}{c *}$ reflects that how much cheaper the cost-optimal path is compared with the fastest path. In Fig. 10(a), we study $\frac{c^{\prime}}{c *}$ by varying the length of time interval $\left[t_{d}, t_{a}\right]$. In this group of experiments, the earliest departure time is fixed to time point 0 and the latest arrival time ranges from time point 300 to time point 1200 . We find $\frac{c^{\prime}}{c *}$ increases with the length of time interval $\left[t_{d}, t_{a}\right]$ increasing. When time interval is $[0,1200], \frac{c^{\prime}}{c *}$ is larger than 6 . It indicates the fastest path is at least six times more expensive than the cost-optimal path. In Fig. 10(b), we study $\frac{c^{\prime}}{c *}$ by varying the distance between source and destination. We also find $\frac{c^{\prime}}{c *}$ increases with the increasing of distance. These experimental results show that the fastest path may not be a cost-optimal path and it is always much more expensive than a cost-optimal path.

\section{RELATED WORK}

Shortest path query is an important problem in graphs and has been well studied in static graphs. The existing works for the shortest path problem propose various index techniques to enhance the efficiency of the shortest path query for large graphs [23, 1, 8, 22, $5,19]$. All these works make a trade-off between the querying time 
and index size. The main idea of these works are maintaining some shortest paths in index. Given a query, algorithms first retrieve the shortest path and then concatenate them by the shortest paths not in index. The recent literature [20] gives a full overview of the works on the shortest path problem.

Recently, there are several works on the shortest path problem in time-dependent graphs. However, these works are to solve the TDSP problem, that is, to find a path from source to destination that has the minimum travel time. These works are categorized as follows: one group is based on the discrete time model and the other one is based on the continuous time model. George et al. in [10] and [9] assign an aggregated attribute to each edge, called edge time series, which represents the time point at which the edge appears. Chabini et al. in [4] discretize the starting-time interval into $k$ time points evenly, and construct a static graph by making $k$ copies of each node and each edge, respectively. The TDSP problem can be solved as a static single-source shortest path problem in such a static graph, whose size is enlarged $k$ times. The fundamental drawbacks of the discrete time model are two-fold. First, it cannot represent the state of networks between two discrete time points, which might yield inaccurate results. Second, the memory and processing requirement are high. Orda et al. propose a continuous time algorithm to solve the TDSP problem in [16]. This algorithm generalizes the BELLMAN-FORD shortest path algorithm. Sung et al. [21] present a Flow Speed Model that computes the travel time on each road segment as a piecewise linear function of time. Pfoser et al. [17, 18] contribute techniques that can derive the up-to-date speed associated with a road segment at a given time based on Floating Car Data. Kanoulas et al. in [13] propose an $\mathrm{A}^{*_{-}}$ extended algorithm. The main idea of this algorithm is to maintain a priority queue $Q$ of all the paths to be expanded. For any vertex $v_{i}$, the algorithm estimates the travel time from $v_{i}$ to destination $v_{e}$. By estimating the travel time, the algorithm computes a shortest path from source to destination with the minimum travel time. Gonzalez et al. in [11] apply some data mining techniques to derive driving and speed patterns that describe road speeds under a variety of conditions, such as time, weather, and vehicle type. Ding et al. in [7] propose an efficient $2 \mathrm{~S}$ algorithm to solve the TDSP problem. All these methods utilize the following property: the earliest arrival time of a vertex $v_{i}$ can be computed by the earliest arrival time of $v_{i}$ 's incoming neighbors. However, for the problem proposed in this paper, this property does not hold. Therefore, the solution to the TDSP problem cannot be used to solve the cost-optimal path problem proposed in this paper.

Several studies in the field of operation research consider the cost-optimal path problem under the discrete time model $[3,2]$. In the discrete time model, the whole time interval is discretized to a set of time points, $\left\{t_{1}, t_{2}, \cdots, t_{l}\right\}$. For any edge $\left(v_{i}, v_{j}\right)$, users only can select a specified time point $t_{x}$ to depart from $v_{i}$. The main disadvantages of these works are as follows: (1) The optimal path cannot be found under the discrete time model. (2) These works need to compute the arriving cost for every vertex at every time point. The time and space costs are expensive.

\section{CONCLUSION}

In this paper, we study how to find a cost-optimal path with time constraint in time-dependent graphs. We first define the costoptimal path with time constraint. Second, we propose an efficient TWO-STEP-SEARCH algorithm to compute a cost-optimal path with time constraint. We show that the time and space complexities of the algorithm are $O(k n \log n+m k)$ and $O((n+m) k)$ respectively. Finally, we confirm the effectiveness and efficiency of our algorithm through conducting experiments on real datasets.

\section{ACKNOWLEDGMENTS}

This work is partly supported by the grant of the National Program on Key Basic Research Project (973 Program) of China, No. 2012CB316200, the grant of the National Natural Science Foundation of China No. 61190115, 61173022, and the grant of the Research Grants Council of Hong Kong SAR, No. CUHK 418512.

\section{REFERENCES}

[1] T. Akiba, Y. Iwata, and Y. Yoshida. Fast exact shortest-path distance queries on large networks by pruned landmark labeling. In SIGMOD Conference, pages 349-360, 2013.

[2] X. Cai, T. Kloks, and C. Wong. Time-varying shortest path problems with constraints. Networks, 29(3):141-150, 1997.

[3] X. Cai, T. Kloks, and C. K. Wong. Shortest path problems with time constraints. In MFCS, pages 255-266, 1996.

[4] I. Chabini. Discrete dynamic shortest path problem in transportation applications: Complexity and algorithms with optimal run time. Transportation Research Records, 1645:170-175, 1998.

[5] J. Cheng, Y. Ke, S. Chu, and C. Cheng. Efficient processing of distance queries in large graphs: a vertex cover approach. In SIGMOD Conference, pages 457-468, 2012.

[6] T. H. Cormen, C. E. Leiserson, R. L. Rivest, and C. Stein. Introduction to Algorithms, Second Edition. The MIT Press, 2001.

[7] B. Ding, J. X. Yu, and L. Qin. Finding time-dependent shortest paths over large graphs. In EDBT, pages 205-216, 2008.

[8] J. Gao, R. Jin, J. Zhou, J. X. Yu, X. Jiang, and T. Wang. Relational approach for shortest path discovery over large graphs. In $P V L D B$, volume 5, pages 358-369, 2012.

[9] B. George, S. Kim, and S. Shekhar. Spatio-temporal network databases and routing algorithms: A summary of results. In SSTD, pages 460-477, 2007.

[10] B. George and S. Shekhar. Time-aggregated graphs for modeling spatio-temporal networks. Journal on Data Semantics, 11:191-212, 2006.

[11] H. Gonzalez, J. Han, X. Li, M. Myslinska, and J. P. Sondag. Adaptive fastest path computation on a road network: A traffic mining approach. In $V L D B$, pages 794-805, 2007.

[12] L. Jiang, S. Parekh, and J. Walrand. Time dependent network pricing and bandwidth trading. In NOMS Workshops, pages 193-200, 2008.

[13] E. Kanoulas, Y. Du, T. Xia, and D. Zhang. Finding fastest paths on a road network with speed patterns. In ICDE, pages 10-19, 2006.

[14] J. Leape. The london congestion charge. Journal of Economic Perspectives, 20(4):157-176, 2006.

[15] M. O’Mahony, W. Y. Szeto, and X. Q. Li. Modeling time-dependent tolls under transport, land use, and environment considerations. In Applications of Advanced Technology in Transportation, pages 852-857, 2006.

[16] A. Orda and R. Rom. Shortest-path and minimum-delay algorithms in networks with time-dependent edge-length. Journal of ACM, 37(3):607-625, 1990.

[17] D. Pfoser, S. Brakatsoulas, P. Brosch, M. Umlauft, N. Tryfona, and $\mathrm{G}$. Tsironis. Dynamic travel time provision for road networks. In GIS, pages 68-71, 2008.

[18] D. Pfoser, N. Tryfona, and A. Voisard. Dynamic travel time maps enabling efficient navigation. In SSDBM, pages 369-378, 2006.

[19] M. Qiao, H. Cheng, L. Chang, and J. X. Yu. Approximate shortest distance computing: A query-dependent local landmark scheme. In ICDE, pages 462-473, 2012.

[20] C. Sommer. Shortest-path queries in static networks. ACM Computing Surveys, 46(4):1-35, 2014.

[21] K. Sung, M. G. Bell, M. Seong, and S. Park. Shortest paths in a network with time-dependent flow speeds. European Journal of Operational Research, 121(1):32-39, 2000.

[22] L. Wu, X. Xiao, D. Deng, G. Cong, A. D. Zhu, and S. Zhou. Shortest path and distance queries on road networks: An experimental evaluation. PVLDB, 5(5):406-417, 2012.

[23] A. D. Zhu, H. Ma, X. Xiao, S. Luo, Y. Tang, and S. Zhou. Shortest path and distance queries on road networks: towards bridging theory and practice. In SIGMOD Conference, pages 857-868, 2013. 